Running head: Rumination and reinforcement learning

\title{
Rumination Derails Reinforcement Learning with Possible Implications for Ineffective Behavior
}

Peter Hitchcock, ${ }^{1}$ Evan Forman, ${ }^{2}$ Nina Rothstein, ${ }^{3}$ Fengqing Zhang, ${ }^{2}$ John Kounios, ${ }^{3}$ Yael Niv,${ }^{4 *}$ Chris Sims ${ }^{5 *}$

1 Cognitive, Linguistic, and Psychological Sciences, Brown University, Providence, RI 2 Psychology Department, Drexel University, Philadelphia, PA

3 Applied Cognitive \& Brain Sciences, Drexel University, Philadelphia, PA

4 Princeton Neuroscience Institute \& Psychology Department, Princeton University, Princeton, NJ 5 Cognitive Science Department, Rensselaer Polytechnic Institute, Troy, NY

* Equally contributing authors

Corresponding author:

Peter F. Hitchcock

Department of Cognitive, Linguistic, and Psychological Sciences

Brown University

Providence, RI 02912

Email: peter_hitchcock@brown.edu

Citation:

Hitchcock, P., Forman, E., Rothstein, N., Zhang, F., Kounios, J., Niv, Y., ${ }^{*}$ Sims, C. (in press). Rumination Derails Reinforcement Learning with Possible Implications for Ineffective Behavior. Clinical Psychological Science. https://doi.org/10.1177/21677026211051324 


\begin{abstract}
How does rumination affect reinforcement learning - the ubiquitous process by which we adjust behavior after error in order to behave more effectively in the future? In a within-subject design $(n=49)$, we tested whether experimentally manipulated rumination disrupts reinforcement learning in a multidimensional learning task previously shown to rely on selective attention. Rumination impaired performance, yet unexpectedly this impairment could not be attributed to decreased attentional breadth (quantified using a "decay" parameter in a computational model). Instead, trait rumination (between subjects) was associated with higher decay rates (implying narrower attention), yet not with impaired performance. Our task-performance results accord with the possibility that state rumination promotes stress-generating behavior in part by disrupting reinforcement learning. The trait-rumination finding accords with the predictions of a prominent model of trait rumination (the attentional-scope model). More work is needed to understand the specific mechanisms by which state rumination disrupts reinforcement learning.

\section{Keywords:}

Rumination, Reinforcement learning, Adaptive Behavior, Attention, Computational modeling, Computational psychiatry 


\section{Introduction}

Rumination-thinking passively and abstractly about one's distress and its possible implications-is a potent, transdiagnostic risk factor for mental health problems, especially depression and anxiety disorders (Nolen-Hoeksema, Wisco, \& Lyubomirsky, 2008; Watkins \& Roberts, 2020). Unipolar depression and anxiety disorders in turn increase mortality risk by an estimated 108\% and 43\% respectively (Walker, McGee, \& Druss, 2015) and account for more than twice the daily adjusted living costs of other mental disorders (National Institutes of Mental Health, 2015). Meta-analysis has shown that rumination correlates more strongly with these disorders than several other well-studied response styles (Aldao, Nolen-Hoeksema, \& Schweizer, 2010). Longitudinally, rumination predicts future depression among initially non-depressed individuals (e.g., Abela, Brozina, \& Haigh, 2002) and later depression among already-depressed individuals after controlling for baseline symptoms (e.g., Kuehner \& Weber, 1999), and mediates the relationship between baseline depression and future anxiety (McLaughlin \& Nolen-Hoeksema, 2011). Conversely, treating rumination holds promise for preventing later depression and anxiety (Cook, Mostazier, \& Watkins, 2019). Thus, rumination appears to play a critical role in these deadly and costly disorders.

Rumination may lead to mental-health problems in part by promoting ineffective behavior that generates stress and ultimately harms mental health. Rumination is associated crosssectionally with behaviors that appear likely to cause problems and generate stress, including dependence (e.g., Spasojević \& Alloy, 2001), submissiveness (Pearson, Watkins, Mullan, \& Moberly, 2010), decreased warmth (e.g., Tester-Jones, Karl, Watkins, \& O'Mahen, 2017), avoidance of challenge (Moulds, Starr, and Wong, 2007), low goal attainment (Moberly \& Watkins, 2010), reckless behavior (Nolen-Hoeksema \& Morrow, 1991), impaired cognitive performance (e.g., Jones, Siegle, Muelly, Haggerty, \& Ghinassi, 2010), and poor problem solving in relationships (e.g., Grimbos, Granic, Pepler, 2013). Longitudinally, rumination predicts problematic behavior and dependent social stress, such as aggression and perceived 
victimization, which in turn predict depression and anxiety symptoms (McLaughlin, Aladao, Wisco, \& Hilt, 2014; McLaughlin \& Nolen-Hoeksema, 2014). Theorists propose that ineffective behavior and dependent stress arise as ruminative individuals fail to act effectively when navigating challenging situations (e.g., Kuhl, 1981; Lyubomirsky \& Tkach, 2004; Nolen-Hoeksema, 1987; Snyder \& Hankin, 2016).

One prominent way to learn what is (and what is not) effective is through reinforcement learning $(R L)$ : the process through which we adjust expectations and actions when an outcome differs from what we expected (Sutton \& Barto, 2018). When a joke misses the mark, a consolation fails to soothe, or an attempt to elicit support goes unrequited, RL allows us to adapt so that we act more effectively in the future. The ubiquity of RL makes it critical to healthy behavior. If rumination systematically interferes with $\mathrm{RL}$-even in a relatively minor way-it could disrupt adaptive behavior (Maia \& Frank, 2011). Possibly, this interference could eventually help to explain why rumination is associated with such a diverse array of ineffective behaviors.

One reason to think that rumination may disrupt $\mathrm{RL}$ is that various psychotherapies aim to counteract rumination's effects on learning. For instance, the Behavioral Activation (BA) for depression manual notes that unconstructive repetitive negative thinking can block the effects of behavioral assignments, which are designed to promote learning and increase contact with reward, by keeping individuals so preoccupied that they fail to engage during these experiences (Martell, Dimidjian, \& Herman-Dunn, 2013). Acceptance and Commitment Therapy (ACT) teaches skills—such as present-moment awareness, mental distance ("defusion”) from thoughts, and monitoring of self-referential content-to increase contact with environmental contingencies related to valued domains (Hayes, Strohsahl, \& Wilson, 2011; Herbert, Hitchcock, \& Forman, 2016). Thus, ACT, BA, and other psychotherapies emphasize that repetitive negative thinking such as rumination can disrupt experiences that might otherwise pave the way to mental health recovery. Yet, the field lacks a unifying basic science framework for understanding the effects of these techniques across psychotherapies (see also, Reilly et al., 2019). 
Broadly consistent with the possibility that rumination impairs attention to contingencies relevant to adaptive behavior, past research has suggested that rumination is associated with reduced sensitivity to contextual details (Stein, Lehtonen, Harvey, Nicol-Harper, \& Craske, 2009; Watkins, 2008; Watkins \& Roberts, 2020). For instance, experimental rumination manipulations lead mothers who are dysphoric (Tester-Jones, Karl, Watkins, O'Mahen, 2016) or diagnosed with Major Depressive Disorder or Generalized Anxiety Disorder (Stein et al., 2012) to be less responsive to infants, and diminishes the ability of female undergraduates who endorse at least moderate preoccupation with eating, weight, and body shape issues to recall the emotional details of a video showing a family interaction (Lehonten et al., 2009).

One past experimental rumination-manipulation study specifically showed that rumination alters learning in in an RL setting (Whitmer, Frank, \& Gotlib, 2012). This study suggested that rumination disrupts long-term learning to avoid punishing (but not select rewarding) stimuli. Our study builds on this work while incorporating a within-subject design and using a task that more strongly captures the interaction of higher-order cognitive processes with the core RL system. This latter design choice is important because rumination is believed to interfere with cortical functions (e.g., Jones et al., 2010; Philippot \& Brutoux, 2008), suggesting that it is more likely to interfere with this interaction than with the operation of the (primarily subcortical, evolutionarily conserved) core RL system per se. Indeed, Whitmer et al. (2012) speculated that their findings of disrupted long-term punishment learning could have arisen from enhanced cortical representations of punishing contingencies during rumination, although they were unable to pinpoint such effects. Much research since has emphasized the importance of higher-order cognitive systems in guiding adaptive human RL (reviewed in Niv, 2019; Rsmus, McDougle, \& Collins, 2021)—and these interactions' relevance to mental health (Radulescu \& Niv, 2019)— underscoring that disruption to these interactions could promote ineffective behavior and ultimately poor mental health. Finally, while Whitmer et al. (2012) focused on the effects of state rumination on $\mathrm{RL}$, trait rumination has been associated with altered functioning of cognitive 
systems that could plausibly influence RL and notably may have distinct effects from those caused by experimentally manipulated rumination (Whitmer \& Gotlib, 2013). Thus, we measure both state and trait rumination in the current study.

To further understand if and how rumination alters RL, we conducted a within-subject investigation with a relatively large sample $(n=49)$. Participants performed a challenging trial-anderror learning task designed to measure the cooperative interplay of selective attention (a higherorder cognitive process) with the RL system (from Niv et al., 2015) while receiving a manipulation designed to instigate short bouts of ruminative thinking (versus neutral thinking). A large literature suggests rumination hijacks attentional systems (e.g., Hamilton et al., 2011; Kaiser, AndrewsHanna, Wager, \& Pizzagalli, 2015), making it plausible that bouts of rumination would disrupt the cooperative interaction of attention and $R L$.

Because we were especially interested in how the intrusion of ruminative thought might lead to temporary—yet systematic and cumulatively important—disruptions in the ability to adapt to and learn from contingences, we adapted a classic rumination induction procedure (NolenHoeksema \& Morrow, 1993) and repeatedly exposed participants to cues designed (in the rumination condition) to instigate brief bouts of ruminative thinking, interleaved with their performing the trial-and-error learning task. Although it is unclear whether our procedure leads to sustained rumination in the manner of the traditional procedure (see Hertel \& Rude, 1991), we chose this design to create an experimental analogue of real-life situations where a cue to ruminate (such as perceiving that one has committed a faux pas while socializing with colleagues) may create a short-lived disruption in the ability to adapt to, and learn about, contingencies that could allow for more adaptive behavior both in the moment and in the future.

We expected that the experimental rumination manipulation would impair RL task performance due to lower attentional breadth for representing task features while learning—which can be quantified in our task using a computational model parameter: the "decay" of learned information about unattended aspects of the task (Niv et al., 2015). In addition to experimentally 
manipulating rumination (within subject) we examined the effects of trait rumination (between subjects) on performance and model parameters.

Consistent with our first hypothesis, but contrary to our second, we found that experimentally manipulated rumination was associated with worse task performance, yet not with a higher decay parameter. One caveat is that a manipulation check that relied on participants' subjective reports found only weak evidence that the experimental manipulation induced rumination (at least as evident at the time of the manipulation checks). Conversely, trait rumination was associated with a higher decay parameter, but not with significantly altered task performance. These findings support the possibility that state rumination disrupts $\mathrm{RL}$ in everyday life, and that this disruption may partly underpin the associations between rumination and various forms of ineffective behavior. The results further suggest that state and trait rumination have distinct effects on RL.

\section{Methods}

\section{Participants}

We recruited 56 adult undergraduate participants (age 18-34; $M=19.96, S D=2.30$ ) at Drexel University with elevated depression symptoms, specifically, a Beck's Depression Inventory-II (Beck, Steer, Brown, 1996) sum score of at least 14. Participants identified as: 73.21\% female, $26.79 \%$ male; $8.93 \%$ Hispanic and the remainder non-Hispanic; $10.71 \%$ African American, 33.93\% Asian American, 10.71\% Middle Eastern, 35.71\% European American, 1.8\% Native American, $5.36 \%$ Multiracial, and $1.79 \%$ other; and $33.93 \%$ non-English-speaking as a first language, with 5 the median age at which the non-native-English speakers began learning English. All participants reported having never been diagnosed with a major neurological illness. 


\section{Materials}

\section{Self-report}

\section{Rumination and Response Scale-Short Form (RRS-SF)}

Trait rumination was assessed using the short-form version of the rumination scale of the Response Styles Questionnaire (Nolen-Hoeksema \& Morrow, 1991). The RRS-SF (Treynor, Gonalez, \& Nolen-Hoeksema, 2003) excludes items from the original scale that overlap with depression symptoms, leaving 10 items, 5 of which are grouped into a "reflective pondering" subscale (e.g., "Go someplace alone to think about your feelings") and 5 into "brooding" (e.g., “Think 'Why can't I handle things better?'”) subscales. Items are rated on a scale from 1-4 ranging from "I almost never respond this way" to "I almost always respond this way." Treynor et al. (2003) provided evidence of the internal consistency and construct validity of the measure. We chose this commonly used measure to facilitate comparison with previous studies examining how rumination relates to experimental tasks (Whitmer \& Gotlib, 2013). Internal consistency as assessed by Cronbach's $\alpha$ was somewhat lower in this sample for both subscales (brooding=.62, reflect $=.53$ ) than previously reported in the literature (e.g., brooding=.77, reflect=.72 in Treynor et al., 2003).

\section{Beck's Depression Inventory-II (BDI-II)}

Depression symptoms were assessed using the BDI-II, a 21-item assessment of depression symptoms. Items are rated from 0 to 3 with higher scores corresponding to more severe symptoms. The BDI-II has shown adequate reliability and validity in prior research (Titov et al., 2011) although it (and other depression measures) have also been criticized for lack of construct validity due to the heterogeneity of depression symptoms (Fried \& Nesse, 2015). We employed this measure as a screening tool for dysphoria for consistency with many past rumination-manipulation studies (reviewed in Lyubomirsky, Layous, Chancellor, \& Nelson, 2015). Internal consistency as assessed by Cronbach's $\alpha$ was again somewhat lower in our sample (.66) than previously reported in the literature (e.g., .87-.90 in Titov et al., 2011). We discuss the lower 
internal consistency (as per Cronbach's $\alpha$ ) of the RRS-SF and BDI-II in this sample in discussing the limitations of the study (see Discussion).

\section{Manipulation Check Effectiveness Measure}

To test if our rumination manipulation elevated state rumination (relative to a neutral condition), participants answered manipulation-check questions before each manipulation condition and then after being exposed to it (see "Procedure"). At the start of the study, we were unaware of any existing measures of state rumination, hence we created our own by asking participants to make 11 ratings on a Likert scale of 1-9 about their current positive and negative affect and self-referential thinking (see Methodological Details S1 in Supplemental Material available online for the list of items). Past studies showed that rumination increases negative affect and self-referential thinking and decreases positive affect (e.g., Ciesla \& Roberts, 2007; Kaiser et al., 2018; McLaughlin, Borkovec, \& Sibavra, 2007; Moberly \& Watkins, 2006), hence we reasoned that if the rumination manipulation were effective, scores on these items should increase relative to the neutral condition. We reverse-scored positive-affect items and then took the average of the manipulation-check items as a state measure of manipulation effectiveness, with higher scores indicating greater state rumination. Item order was counterbalanced across participants and half of the items were presented with the anchor flipped (e.g., "Focused on myself" on the left versus right side of the screen) to promote careful responding. Internal 

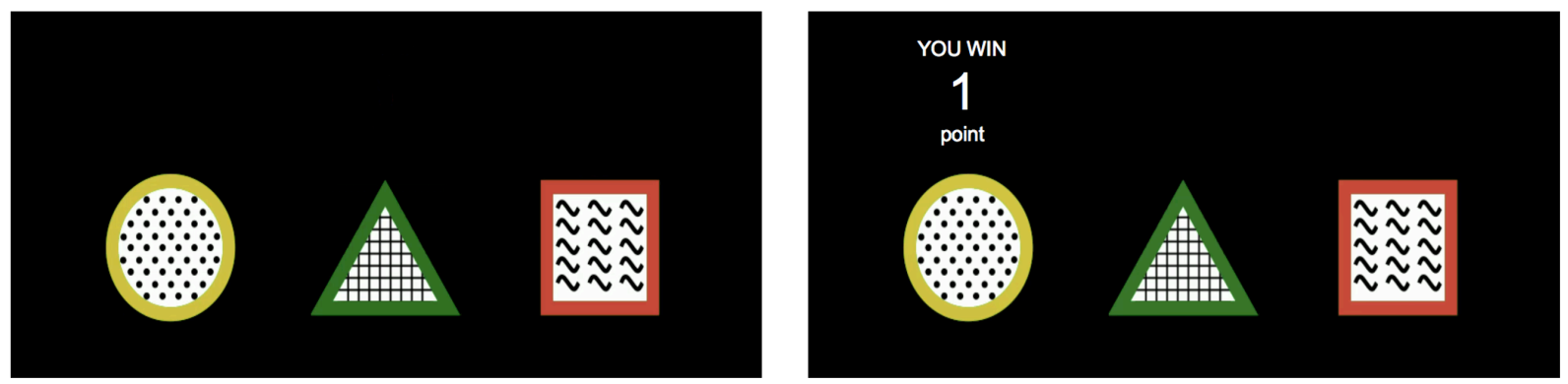

Figure 1. The Dimensions Task as it appears to participants before (left) and after (right) a choice.

consistencies of the manipulation check items as assessed by Cronbach's $\alpha$ at baseline and postmanipulation were .85 and .82 respectively.

\section{Behavioral Task}

The Dimensions Task is a multidimensional $\mathrm{RL}$ task that investigates the interaction between trial-and-error learning and attention (Leong, Radulescu, et al., 2017; Niv et al., 2015; Radulescu, Daniel, \& Niv, 2016; Daniel et al., 2020). On each trial participants chose one of three stimuli and received feedback (Figure 1). Each stimulus consisted of three features in each of three dimensions: color (yellow, green, or red), texture (dots, plaid, or waves), and shape (circle, triangle, or square), such that all nine features appeared in each trial.

Participants played "games" comprising sequences of 30 trials. In a given game one feature was covertly designated the "target" feature; this meant that selecting the stimulus with that feature resulted in reward with high probability $(p=.75)$ whereas selecting any other stimulus led to reward with low probability $(p=.25)$. Participants were neither told what the target feature was nor what dimension (color, shape or texture) it was in and had to learn this by trial and error to maximize reward. The target feature changed with each game; each new target was drawn from a different dimension than the prior game. Game changes were explicitly signaled ("Game starts in 3 seconds..." message on the screen).

The reward outcome ( 1 point or 0 points) was presented above the chosen stimulus for $500 \mathrm{~ms}$ immediately following choice (Figure 1, right). The inter-trial interval was $300 \mathrm{~ms}$ and 
participants received time-out feedback if they did not select a stimulus within $2 \mathrm{~s}$ of presentation of the stimuli on screen. Participants were trained on the task prior to commencing the experiment to ensure they understood the task structure and instructions (i.e., there is one target feature in each game and the high versus low probabilities of reward given choice of stimulus) and were instructed to try to maximize their point rewards (see "Study Visit" for further details). Behavioral performance was calculated as the proportion of trials on which participants selected the stimulus with the target feature.

The Dimensions Task is thought to engage both selective attention and RL because each game involves one informative dimension (e.g., color) and two "distractor" dimensions. Selectively attending to the dimension of the target feature (e.g., trying to determine which color is most rewarding while ignoring shape and texture) can facilitate learning. Past work showed that $R L$ and choice are both constrained by dimensional attention that changes throughout the game (Leong, Radulescu, et al., 2017).

\section{Procedure}

\section{Eligibility Screening}

Using the procedure from many past rumination-manipulation studies (reviewed in NolenHoeksema et al., 2008), we recruited participants with elevated depression scores (BDI-II $\geq 14$ ) via eligibility screening questionnaires comprising the BDI-II and a measure of perfectionism (the latter included only to blind participants to the criterion of elevated depression symptoms). Onehundred-and-thirty-six (of 339) undergraduate students reported BDI-II scores of at least 14 . The

first and third authors (P.H. and N.R.) screened questionnaire responses of low quality (e.g., completion in less than a minute, all questions with the same answer); participants reporting high suicidal ideation (via a score of 3 on question 9 of the BDI-II) were also excluded. Eligible participants were invited to participate in the in-person study and were required to complete the visit within 2 weeks of the eligibility screening (Lyubomirsky \& Nolen-Hoeskema, 1993). 


\section{Rumination and Neutral Manipulation}

At study visit, participants received an adaptation of a well-studied induction procedure (e.g., Lewis, Blanco, Raila, \& Joormann, 2019; Nolen-Hoeksema \& Morrow, 1993). This entailed exposing participants to cues designed to instigate either ruminative (RUM) or a neutral (NEU) thinking. The heading "THINK ABOUT" was shown above both sets of cues. The RUM cues (e.g., "your character and who you strive to be"; "why you react the way you do") were designed to provoke abstract self-referential focus and were not intrinsically negatively valenced. As all participants showed elevated dysphoria at screening (and, due to the high test-retest reliability of the BDI in college students, were expected to show elevated dysphoria at the study visit; Lyubomirsky \& Nolen-Hoeksema, 1993), the combination of dysphoria and abstract selfreferential focus was expected to increase state rumination (Watkins, 2008). The NEU manipulation, by contrast, was designed to disrupt the natural state of rumination that participants were expected to be prone to due to dysphoria, thus enabling a contrast of relatively high versus low state rumination. Thus, the NEU manipulation comprised a series of evocative cues (e.g., "a boat slowly crossing the Atlantic"; "clouds forming in the sky") based on the idea that imagining these cues should preoccupy mental resources that would otherwise be devoted to rumination (see Hertel, 1998 and Watkins, 2008 for discussions). The RUM and NEU cues were matched in presentation (e.g., timing, size).

We adapted the procedure used in prior studies in three ways. First, we conducted a within-subjects rather than between-subjects design due to the enhanced precision such designs afford (Gelman, 2018). Second, we recruited only dysphoric participants, as only such individuals were expected to increase state rumination in response to abstract self-referential (but not intrinsically negative) cues on theoretical (Watkins, 2008) and empirical (Nolen-Hoeksema et al., 2008) grounds. Third, whereas past studies typically presented all cues within a single list that participants worked through progressively, and then measured the effect of this manipulation on an outcome variable, we continually cued participants to ruminate in the RUM condition, allowing 
us to examine the effects on learning over an extended period. Thus, we presented 2 cues lasting 10 seconds each prior to the start of each Dimensions Task game (each game lasted for approximately 1 minute); this led to 44 manipulation cues interleaved with 22 games in each condition (see next section). We judged this adaptation important because demanding tasks may eventually pull attention from rumination (Hertel \& Rude, 1991) and because repetitive cues provided a more ecologically valid match to the real-life process we wanted to assay where cues to ruminate (such as a negative thought) may disrupt $\mathrm{RL}$ repeatedly. The amount of time that participants were exposed to each cue (10 seconds) was matched to the exposure time in the classic Nolen-Hoeksema induction paradigm (which includes 45 cues typically completed in selfpaced fashion in 5-8 minutes, that is, $6-11$ s on average per cue).

\section{Study Visit}

Participants provided written informed consent to complete questionnaires and a computerized task and to respond to "visualizations" and "personal cues" (respectively referring to the NEU and RUM conditions). Participants were introduced to the Dimensions Task and the interleaved cues (via an example NEU cue not repeated in the experiment). The introduction included instructions and three practice games: one simplified game where participants were instructed on the dimension containing the target feature (color) and two practice games without instructing the relevant dimension (the relevant dimensions and targets in these games were randomly drawn from any dimension, as in the primary task). Participants then began the experiment throughout which the relevant dimension was never instructed. After each game participants were presented with all features on a screen and asked to select which feature they thought was the target; this continually reminded participants of the task instructions, but these explicit choices were not analyzed. 
There were two experimental phases. Each phase comprised: (1) 22 games (660 trials) ${ }^{1}$ of the Dimensions Task; (2) RUM or NEU cues presented before each Dimensions Task game (condition order was counterbalanced across participants); and (3) manipulation check questions administered at baseline, halfway through the phase (i.e., after exposure to half of the cues), and at the end of the phase (i.e., after exposure to all of the cues). Each phase took approximately 45 minutes. The first phase was followed by a "washout" period lasting about 10 minutes (which was designed to give participants a break from playing the task and to allow the effects of the first manipulation to dissipate); participants walked to an experimental room on a separate floor and completed a questionnaire concerning mindfulness. They then returned to the original experimental room to complete the second phase. Finally, participants completed questionnaires, specifically, the RRS-SF as well as measures of anhedonia, positive and negative affect, cognitive distancing, and dysfunctional attitudes. The latter measures were collected for potential secondary analyses and do not relate to the study's primary purpose, hence they are not discussed further here.

Participants were paid a baseline compensation and a bonus proportional to their RL task performance. Four options were given for baseline compensation: 3 extra credits, $\$ 0 ; 2$ extra credits, $\$ 10 ; 1$ extra credit, $\$ 20$; or no credit, $\$ 30$. Participants were also provided a $\$ 5-\$ 15$ bonus based on their task performance; specifically, we developed expected performance terciles based on performance in a prior study (Radulescu et al., 2016) and compensated participants respectively $\$ 5, \$ 10$, and $\$ 15$ if they reached the bottom, middle, and top terciles. Participants were informed only that they could earn up to a $\$ 15$ bonus for performance.

\footnotetext{
${ }^{1}$ We selected the number of games in each phase based on prior analyses with this task showing that this number facilitated stable within-subject estimation of outcome measures (Hitchcock, Radulescu, Niv, \& Sims, 2017). Establishing this stability was important because low stability of a task measure calls into question whether the measure accurately taps a latent process relevant to mental health (Brown, Chen, Gillan, \& Price, 2020; Hitchcock et al., 2017; Paulus, Huys, \& Maia, 2016; Rodebaugh et al, 2016).
} 


\section{Analyses}

\section{Quality Control}

Adapting criteria from a prior Dimensions Task study (Radulescu et al., 2016, study 2), we excluded from analyses datasets from participants showing near-chance performance in the Dimensions Task in one or both of the RUM or NEU phases. Specifically, criteria for exclusion were (1) if a stimulus containing the target feature was not selected on 6 or more consecutive trials in at least $15 \%$ of games or (2) performing at an overall accuracy of $\leq 37 \%$ correct (within two standard deviations of the mean of a binomial distribution with $p=1 / 3$-i.e., chance performance-and $N=660$-the number of trials in each phase). Forty-nine of 56 participants $(87.50 \%)$ passed these quality control checks. Of these, 24 and 25 participants respectively received the NEU and RUM manipulations first.

\section{Statistical Data Analyses}

Data were preprocessed and analyzed using $\mathrm{R}$ (Version 3.6.2; R Core Team, 2019). For primary analyses, we built ordinary least squares regression models or, for nested data (specifically, manipulation-check and Dimensions-Task behavioral data, which had multiple trials nested within participant), mixed-effects regression models via the Ime4 package (Bates, Mächler, Bolker, \& Walker, 2014) with p-values estimated using Satterthwaite's method for approximating degrees of freedom. We sought to balance the benefits of "maximal" random effect structures (enhanced generalizability and protection against Type I error; Barr, Levy, Scheepers, \& Tily, 2013) with those of parsimonious models (increased power and decreased risk of overparameterization; Bates, Kliegl, Vasishth, Baayen, 2015; Matuschek, Kliegl, Vasishth, Bayeen, \& Bates, 2017). To do this, we first fit maximal random effects structure (full intercept and slope random effects) and then tested whether the model could be made more parsimonious through backward selection via model comparison, that is, by reducing complexity until the loss significantly decreased goodness of fit (Bates et al., 2015; Matsuchek et al., 2017). Model comparison between nested regression models was conducted via the likelihood ratio test 
(Mirman, 2014) with $\alpha=.2$ (following the recommendation of Matuschek et al., 2017, p. 308, for balancing the penalty on model complexity versus goodness of fit in the specific context of mixedeffects regression modeling; note that all results held with $\alpha=.05)$. Collinearity of predictors was assessed via the variable inflation factor (VIF); all predictors had VIF $<1.7$. Details of specific mixed-effects models (e.g., covariates included) are described in "Results." For (paired) comparisons between RUM and NEU where the null hypothesis was of interest, we estimated evidence in favor of the null hypothesis $\left(\mathrm{BF}_{01}\right)$ via the JZS Bayes factor (Rouder, Speckman, Sun, \& Morey, 2009) implemented through the BayesFactor package (Morey, Rouder, Jamil, \& Maintainer, 2015).

\section{Computational Modeling Analyses}

We applied reinforcement-learning computational models to infer latent aspects of learning and choice from task behavior, drawing on an established computational model of the Dimensions task known as feature $R L$ (fRL) + decay (Niv et al., 2015; Radulescu et al., 2016).

This model learned a value for each of the 9 features in the task, $v(f)$. The value of a compound stimulus, $V\left(S_{i}\right)$, was then the sum of its 3 feature values:

$$
V\left(S_{i}\right)=\sum_{f \in S_{i}} v(f)
$$

Feature values were reset to 0 at the start of each game (following instructions to participants that games were independent with a new target feature selected for each game). On each trial, the features of the chosen stimulus were updated according to a prediction error signal—the difference between the reinforcement received, $r$ (either 0 or 1 ), and the value of the chosen stimulus, $V\left(S_{\text {chosen }}\right)$ :

$$
v^{\text {new }}(f)=v^{\text {old }}(f)+\eta\left[r-V\left(S_{\text {chosen }}\right)\right], \text { for all features in the chosen stimulus }
$$

Where $\eta \in[0,1]$, the learning rate, is a free parameter. This update equation reflects incremental updating with higher learning rates resulting in greater updating. 
The values of unchosen features were decayed to 0 on each trial at a rate specified by a free parameter, $d \in[0,1]$ :

$$
v^{\text {new }}(f)=(1-d) v^{\text {old }}(f) \text {, for all features not in the chosen stimulus }
$$

Higher decay rates, $d$, led to unchosen features shrinking toward 0 more quickly. Note that on any given trial, a participant may be selecting a stimulus based on just one or two of its features, not all three.

Not knowing what dimensions the participant was focusing on, our model updates all three features via the prediction error only to "undo" that update on the next trial (through decay) if a feature is not selected again. Because of this, one can view higher decay rates as narrower attention at learning —akin to having learned about some features and not others. Following this intuition and prior work (Niv et al., 2015; Leong, Radulescu et al., 2017), we therefore interpret the $d$ parameter as breadth of attention over features-with lower decay representing greater attentional breadth.

Our model assumes that choices are guided by stimulus values. Intuitively, higher-valued stimuli should have a higher probability of being chosen. To implement this, the model converts values to choice probabilities using the softmax function (an extension of the logistic function for more than 2 options):

$$
p\left(\text { choose } S_{i}\right)=\frac{e^{\beta V\left(S_{i}\right)}}{\sum_{j=1}^{3} e^{\beta V\left(s_{j}\right)}}
$$

Here, $\beta \in[0,40]$ is an inverse-temperature free parameter that determines to what extent highervalued stimuli are favored over lower-valued stimuli. For high $\beta$, choice is more deterministically driven by value differences whereas for low $\beta$ choices are more random.

We also considered an augmented version of the model, $f R L+$ decay with different learning rates $(L R s)$, that allowed different learning rates for the update in equation 2 depending on the sign of the prediction error: $\eta^{-\mathrm{PE}}$ (if prediction error $<0$ ) and $\eta^{+P E}$ (if prediction error $>$ 0 ; both also $\in[0,1])$. 
Procedures for model fitting, model comparison, parameter recovery, and simulations for model validation are described in the Supplemental Material available online.

\section{Results}

\section{Questionnaire Data}

Mean BDI-Il score was $22.51(S D=6.16)$. According to common guidelines for interpreting the BDI-II (Smarr \& Keefer, 2011), 17 (34.69\%) participants fell in the range of mild depression (BDI-II score = 14-19), $24(48.98 \%)$ in the range of moderate depression (BDI-II score = $20-28)$, and $8(16.33 \%)$ in the range of severe depression. Mean rumination score was 29.02 ( $S D=3.89$; $M(\mathrm{SD})$ brooding subscale $=14.92(3.89), M(\mathrm{SD})$ reflect subscale $=14.10(2.63))$. Depression severity showed a low correlation with the brooding subscale $(r(47)=.29, p=.047)$ and no significant correlation with the reflection subscale $(r(47)=.05, p=.716)$.

\section{Manipulation check}

There were no significant differences between manipulation conditions in the manipulation check measure at baseline $\left(p=.473, \mathrm{BF}_{01}=5.03, M(S D)_{R U M}=4.84(1.22), M(S D)_{N E U}=4.93\right.$ (1.14)). The manipulation check measure was differentially elevated by RUM vs. NEU post manipulation only at a trend level in a mixed-effects model that controlled for fixed effects of baseline score (z-scored; thereby controlling for baseline differences by conditions) and manipulation order (controlling for order of administration) and included random intercepts and slopes for baseline score and condition at the subject and manipulation-check-item levels in addition to the "condition" fixed effect of interest $\left(b(S E)=.28(.14), p=.063 ; M(S D)_{R U M}=5.11\right.$ (1.09), $M(S D)_{N E U}=4.97$ (1.11); Table S1 in Supplemental Material available online). A significant elevation in this measure in RUM vs. NEU was present in a simpler model with only subject-level (but not manipulation-check-item-level) random effects $(b(S E)=.24(.11), p=.042)$. Yet, model comparison favored the inclusion of item-level random effects $(\chi(6)=64.12, p<1 \mathrm{e}-11)$ and the inclusion of such effects is conceptually meaningful because the effect of condition and baseline scores could plausibly differ by manipulation check item. Indeed, the condition random effects 
indicated a pattern whereby many (though not all) negative affect and self-referential items showed positive slopes of the RUM condition as expected (indicating elevation relative to the intercept NEU condition), yet the three (reverse-scored) positive affect items showed negative slopes, indicating relatively higher positive affect after the RUM (relative to NEU) manipulation (Table S1 in Supplemental Material available online). Taken together, these results suggest weak evidence per participants' explicit reports that the RUM (vs. NEU) manipulation led to the expected deterioration in affect and increase in self-referential thinking and that these unexpected results were driven in part by a relative increase in reported positive affect after the RUM manipulation.

\section{Effect of Rumination Manipulation and Trait Rumination on Performance}

As predicted, participants selected the correct stimulus on a lower proportion of trials in the rumination relative to neutral manipulation condition, albeit this was a relatively small and heterogenous effect $\left(t_{\text {paired }}(48)=2.49, p=.016, M(S D)_{N E U}=.56(.08), M(S D)_{R U M}=.53(.08)\right.$, Cohen's $d=.33 ; 30 / 49=61.22 \%$ of participants performed worse in RUM than NEU; Figure 2A).

These tests were computed on a summary statistic (proportion correct for each participant in each condition), hence they neither incorporated the trial-wise structure of the data nor controlled for potential confounds. Thus, we confirmed that the performance decrease in the RUM vs. NEU manipulation condition held in a mixed-effects logistic regression model predicting performance according to condition $(b(S E)=-.11(.05), p=.013)$. This model included, as predictors of no interest, fixed effects of trait rumination and depression (z-scored; controlling for the effects of these variables on performance), order of manipulation administration, within-game trial number and within-experimental-phase trial number (rescaled to the range 0 to 1 to improve interpretability; respectively controlling for within-game and within-phase performance change; the latter could occur due to, e.g., fatigue, meta-learning), and random intercepts and slopes at the subject level for condition and the two trial variables (all other covariates were between subject). This model also showed that neither trait rumination $(p=.845$; Figure $2 \mathrm{~B})$ nor depression 
Table 1. Results of task performance mixed-effects logistic regression model.

\begin{tabular}{lcccc}
\hline & $b$ & $\mathrm{SE}$ & $z$ & $p$ \\
\hline Intercept & -.30 & .06 & -5.08 & $<.001^{* *}$ \\
Condition: RUM = 1, NEU = 0 & -.11 & .05 & -2.47 & $.013^{*}$ \\
Rumination (RRS-SF; z-scored) & $<-.01$ & .03 & -.20 & .844 \\
Depression (BDI-II; z-scored) & -.01 & .03 & -.44 & .658 \\
First condition administered: RUM = 1; NEU = 0 & -.07 & .06 & -1.27 & .205 \\
Trial in experimental phase (rescaled to 0-1) & -.03 & .09 & -.30 & .767 \\
Trial in game (rescaled to 0-1) & 1.19 & .08 & 16.28 & $<.001^{* *}$ \\
\hline
\end{tabular}

Note: Fixed effects. ${ }^{* *} p<.01,{ }^{*} p<.05$

A

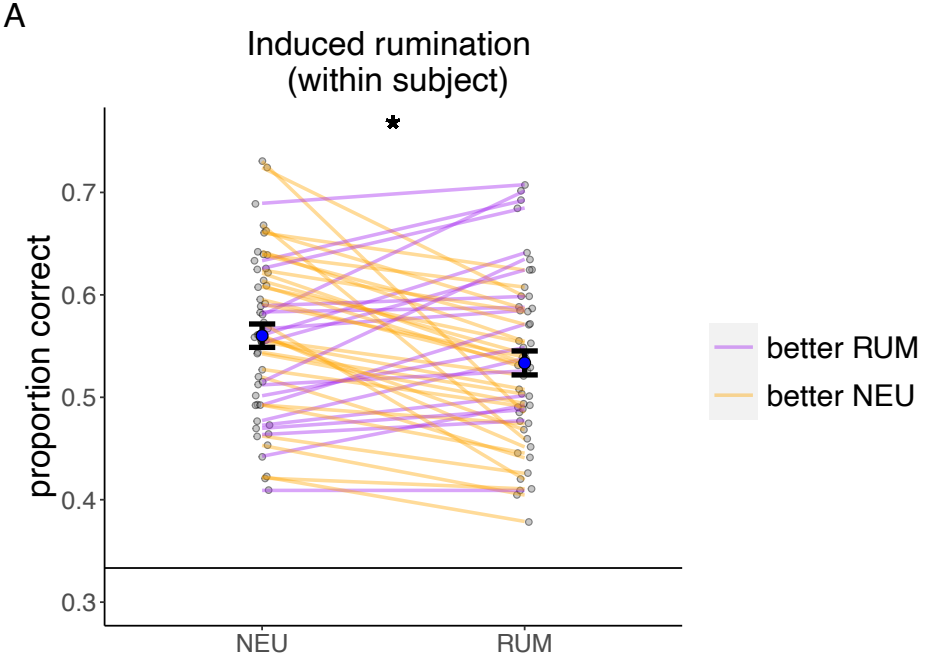

\section{B}

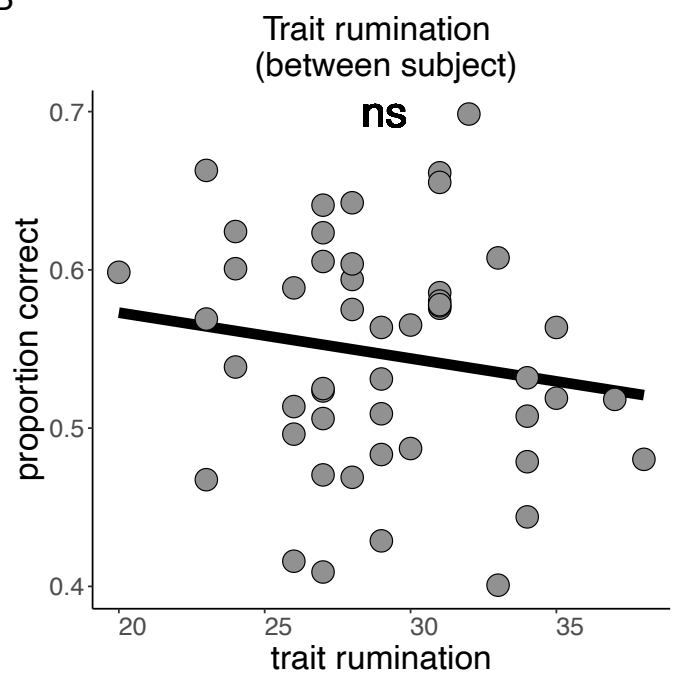

Figure 2. (A) Proportion correct choice (i.e., choice of the stimulus containing the target feature) in the neutral (NEU) and rumination (RUM) experimental manipulation conditions. Blue circles and error bars show mean \pm 1 . S.E.M. Gray circles show subject-level means and lines connect individual subjects with coloring to show relatively better or worse performance in the RUM vs. NEU condition (thus showing the heterogeneity of the effect). (B) Overall proportion correct choices for each participant as a function of trait rumination (with univariate regression line added for illustrative 
$(p=.658)$ had a statistically detectable effect on performance. Model results are presented in Table 1.

In robustness checks, we confirmed that the results were robust to the inclusion of covariates, specifically, that the effect of condition on performance obtained in a model with no fixed effect covariates $(p=.013)$ and with only (z-scored) trait rumination as a covariate $(p=$ .013 ) and that trait rumination still showed no significant relationship with performance in this latter model $(p=.278)$.

\section{Moderation of Performance Effects}

Having found that the RUM manipulation decreased task performance, we next tested whether this effect was moderated by depression severity by including an interaction between condition and depression. The main effect of condition on performance remained significant in this model $(b(S E)=-.11(.04), p=.009)$ and was qualified by a significant interaction between condition and depression severity $(b(S E)=.10(.05), p=.039)$. To clarify this interaction, we constructed follow-up post-hoc regression analyses on subsets of the data comprising participants with higher-than-median and lower-than-or-at-median depression severity levels. A significant effect of manipulation condition on performance was present only among participants with relatively low depression symptoms, whereas there was no statistical evidence for an effect of manipulation condition on performance in those with higher depression symptoms (lower depression: $b(S E)=-.20(.06), p=.002)$; higher depression: $b(S E)=-.02(.06), p=.701)$. Notably, the lower-depression group outperformed the higher-depression group in the neutral condition but performed comparably to them in the rumination condition (NEU: $p=.028 ; M(S D)_{\text {low }}$ depression $=.58(.09), M(S D)_{\text {high depression }}=.53(.06) ; \mathrm{RUM} p=.68 ; \mathrm{BF}_{01}=3.27 ; M(S D)_{\text {low depression }}=$ $\left..54(.08), M(S D)_{\text {high depression }}=.53(.08)\right)$. Thus, the rumination manipulation seemed to cause individuals lower in depression to deteriorate to a comparable performance level as those higher in depression, yet it had little effect on the (already low performing) higher-depression group. 


\section{Model Comparison and Validation}

As in past studies that used the Dimensions Task (Niv et al., 2015), the $f R L+$ decay model fit participants' data considerably better than did a simpler $f R L$ model with no decay rate (range $\left.\Delta B I C_{\text {subject }}=(-115.23,-460.10)\right)$. Thus, we treated $f R L+$ decay, with no splitting of parameters by manipulation condition, as the baseline model. We then iteratively tested whether fit could be improved through alternate models including models where parameters were split (i.e., fit separately) by manipulation condition. $f R L+$ decay with different learning rates with no parameters split provided the best fit to the data (Figure $3 \mathrm{~A}$ ). This suggests that the rumination manipulation did not have a large effect on the learning and choice mechanisms captured by the model parameters; however, there was support for asymmetric rates for learning from positive versus negative prediction errors $\left(\mathrm{BIC}_{\text {subject: }} t_{\text {paired }}(48)=-3.60, p<1 \mathrm{e}-03\right)$. Specifically, there was a substantially lower learning rate from negative than positive prediction errors, suggesting reduced adjustment when outcomes were worse than expected $\left(t_{\text {paired }}(48)=-9.21, p<1 \mathrm{e}-11, \eta^{+P E}: M(S D)\right.$ $\left.=.19(.08), \eta^{-P E}: M(S D)=.09(.06)\right)$. The values of the other parameters in this model were: decay: $M(S D)=.38(.09)$ and inverse temperature: $M(S D)=7.31(5.49)$. Two of the four model parameters were significantly correlated with task performance: higher learning rate from positive prediction errors predicted better performance $(b(S E)=.03(.01), p=.003)$, whereas higher decay rate predicted worse task performance $(b(\mathrm{SE})=-.04(<.01), p<1 \mathrm{e}-3)$; neither inverse temperature nor learning rate from negative prediction errors were significantly associated with performance ( $p$ 's $>.31)$.

Parameter recovery analyses demonstrated excellent ability to recover parameters from this model (Figure 3B). Note that these recoveries used the same number of trials as experienced by participants ( $N=1320$ for each simulated "participant") and data were simulated for parameter values sampled from the range of the parameters' empirical lower and upper bounds. These results show that parameter values can be recovered with high accuracy for this model and with 

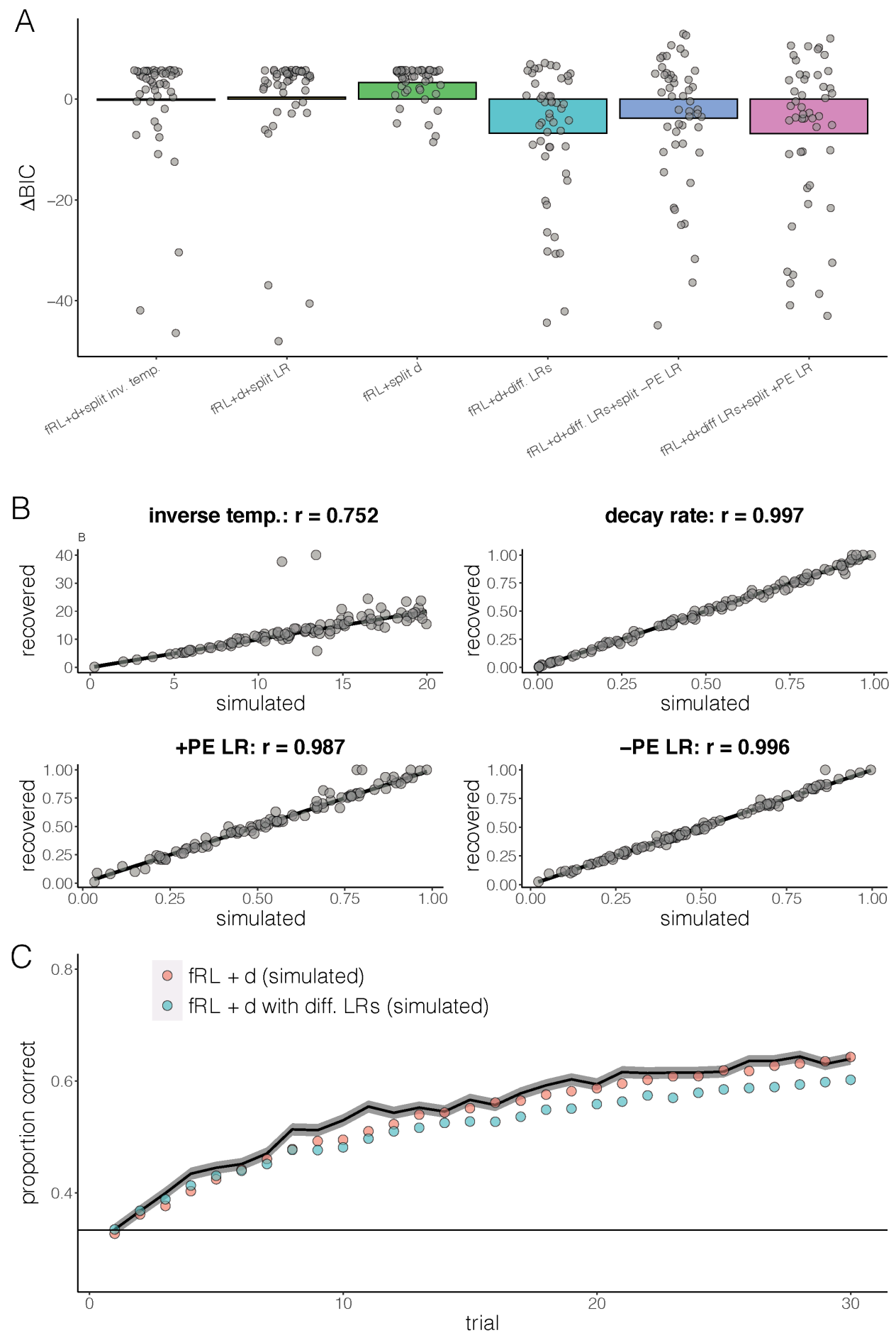

Figure 3. (A) Average (bars) and individual subject (gray circles) difference in BIC for the 6 models considered compared to the baseline model $(f R L+d$ with no parameter splitting). Lower BIC scores are better, so negative values reflect a better fit. Inv. temp. = inverse temperature, $L R=$ learning rate, diff. $=$ different, $d=$ decay, $P E=$ prediction error. (B) Parameter recovery for the most successful model ( $f R L+d$ with different learning rates) shows that parameters are well differentiated and recoverable. $\mathrm{X}$ axis: parameter value used to generate simulated data; $Y$ axis: value of the parameter as fit with maximum likelihood. Circles: each simulated dataset. Solid line is identity line. (C) Simulated learning curves (circles) for comparison with the empirical learning curve (mean: black line; gray error bar: \pm 1 S.E.M.). 
this amount of data. Simulation of learning curves from the $f R L+$ decay and $f R L+d e c a y$ with different learning rates models showed that both models captured the approximate shape of the empirical learning curve, however, both undershot the empirical curve slightly in the early trials, and $f R L+$ decay with different learning rates showed lower asymptotic learning as compared to both the empirical data and the $f R L+$ decay model (Figure $3 C$ ). More specifically, simulations from the $f R L+$ decay model chose the stimulus with the target feature on a significantly smaller proportion of the early-phase (first 15) but not late-phase (last 15) trials compared to the empirical data (early: $p<1 \mathrm{e}-3$; late: $p=.21$ ), whereas the $\mathrm{fRL}+$ decay with different learning rates model was correct on a lower proportion of trials in both phases (early: $p<1 \mathrm{e}-4$; late: $p<1 \mathrm{e}-7$ ). (Comparisons were calculated as paired t-tests on proportion corrects for empirical participants versus simulated "participants.")

Given that these model-validation results were somewhat at odds with the model-selection results, we considered results from both the $f R L+$ decay and the $f R L+$ decay with different learning rates models in the analyses below.

\section{Model Parameters}

The model-comparison results described above provided indirect evidence against our hypothesis that decay rate would increase in the RUM condition because a model with decay rate fit separately by condition (third model in Figure $3 \mathrm{~A}$ ) did not fit the data better than a model with a decay rate that was shared across the RUM and NEU conditions. Given our specific decay-rate hypothesis, we confirmed directly that these parameters did not differ by manipulation condition in the $f R L+$ split decay model $\left(t_{\text {paired }}(48)=0.33, p=.744 ; \mathrm{BF}_{01}=6.12 ; d_{N E U}: M(S D)=.40(.11)\right.$; $\left.d_{P O S} M(S D)=.40(.12)\right)$. Model comparison had also failed to support different rates of learning from negative prediction errors by manipulation condition. Given the past finding suggesting that RUM caused a specific deficit in learning from punishments (Whitmer et al., 2012), we confirmed that the parameter capturing learning from negative prediction errors did not differ by condition in the 


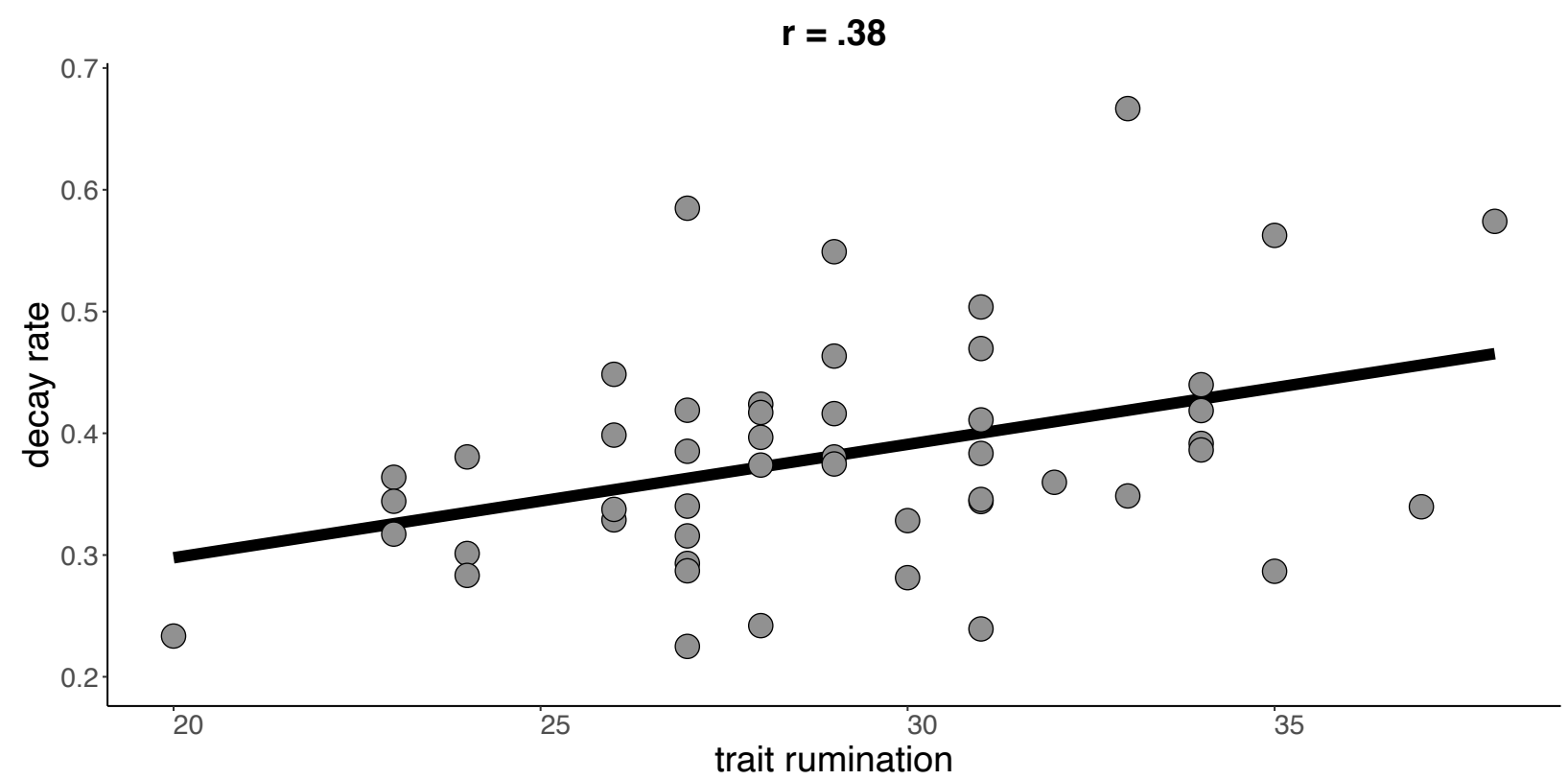

Figure 4. Decay rate relates to with trait rumination Circles: individual participants. Zero-order correlation and univariate regression line added for illustrative purposes.

$f R L+$ decay with different learning rates model $\left(t_{\text {paired }}(48)=0.44, p=.663 ; \mathrm{BF}_{01}=5.88 ; \eta_{N E U}^{-P E}: M\right.$ $\left.(S D)=.09(.06) ; \eta_{R U M}^{-P E} M(S D)=.10(.07)\right)$. Taken together, these results confirm that the mechanisms represented by parameters in our models were unaffected by the experimental rumination manipulation.

In contrast, in a multivariate linear regression of trait rumination on (z-scored) model parameters from the $f R L+d e c a y$ with different learning rates model, trait rumination was positively associated with decay $(b(S E)=1.41(.57), p=.018$; Figure 4$)$. That is, trait-ruminative individuals had lower attentional breadth as per this parameter. No other parameters related to trait rumination ( $p$ 's $>.46)$. In robustness checks, the positive association between trait rumination and decay obtained without the other model parameters as covariates $(b(S E)=1.49(.52), p=.007)$ and in the $f R L+$ decay model with and without the other model parameters as covariates (with: $b(S E)=1.33(.60), p=.030$; without: $b(=S E)=1.44(.53), p=.009)$. Interestingly, regressing the different subscales of the RRS-SF instrument on $f R L+$ decay with different learning rates model 
parameters in separate regression models showed no significant relationship between the brooding subscale and decay rate and a trending relationship between the reflection subscale and decay rate (brood: $b(S E)=.64(.44), p=.154 ;$ reflect: $b(S E)=.77(.40), p=.061)$.

\section{Discussion}

Some people expend significant time and mental energy trying to discern the meanings and implications of their problems, experiences, and personality—that is, ruminating. Rumination appears to predict and perpetuate mental unhealth, with an especially strong link to depression and anxiety disorders. We suspected that one reason for the apparently pernicious effects of rumination on mental health is that rumination disrupts reinforcement learning (RL) - the ubiquitous process of trial-and-error learning that enables healthy behavior. Indeed, we found that experimentally manipulated rumination disrupted performance in a challenging task that requires the cooperative interaction of $\mathrm{RL}$ and selective-attention systems (Leong, Radulescu, et al., 2017). Contrary to our hypothesis, however, this manipulation did not influence the breadth of attention in trial-and-error learning, as quantified by a "decay" parameter in our computational model of behavior on the task. We did find that trait rumination was associated with higher decay and (hence narrower attentional scope in this RL context), but not with disrupted task performance. An important limitation of our study is that we found only weak evidence that the experimental rumination manipulation was effective (although there are some challenges in interpreting the manipulation-check measure we used to make this assessment, as we discuss below). Overall, our findings accord with the idea that experimentally manipulated rumination disrupts RL, raising the possibility that such disruption partly underpins the diverse array of maladaptive behaviors that have been linked to rumination (reviewed in Watkins, 2008). Moreover, the trait-rumination finding supports a prominent theory suggesting that trait rumination partly reflects a cognitive style relating to low attentional scope (Whitmer \& Gotlib, 2013). We discuss each result in turn, beginning with the experimental manipulation finding. Hereafter, we refer to the experimental manipulation effects as state rumination effects (because these involve 
effects experimentally manipulated within subject), whereas effects related to trait rumination refer to findings concerning between-subjects individual differences assessed via the trait rumination questionnaire (RRS-SF).

Nolen-Hoeksema (1987) recognized that rumination may cause mental health problems in part by promoting ineffective behavior, noting that rumination may "focus the individual's attention to his or her emotional state and inhibit any actions that might distract the individual from his or her mood" (Nolen-Hoeksema, 1991, p. 569; emphasis ours). Our findings do not speak to whether rumination inhibits the performance of already-learned actions but suggest an additional mechanism through which state rumination may impede effective behavior, namely, by disrupting ongoing learning. Disrupted RL is possibly more insidious than inhibited action; it not only affects the ability to take effective action during ongoing experience but also has prolonged effects on subsequent experiences that would have benefited from learning had it taken place (cf. Reilly et al., 2019). Thus, the results of this study demonstrate that state rumination may disrupt $\mathrm{RL}$, suggesting it may impair effective action in the moment (due to failure to adapt to errors during ongoing experience) and lower the chance of adaptive actions in similar situations in the future.

In parallel to these effects on behavior, rumination may modify memories so as to increase the likelihood of ruminating again in the future. Specifically, because rumination by definition involves repeatedly bringing negative memories to mind (Nolen-Hoeksema et al., 2008), it may lead to schematization (loss of contextual detail) of such memories-leaving them in a broad template format applicable to many future situations (Audrain \& McAndrews, 2020). Rumination may also strengthen associations among such decontextualized (Williams, 1996) and negative (Cohen \& Kahana, 2019; Grant, Mills, Judah, \& White, 2019; van Vugt, Hitchcock, Shahar, \& Britton, 2012; van Vugt et al., 2018) memories. Watkins (2008) reviewed evidence suggesting that low-detail, highly general memories are unhelpful to problem solving and generate negative affect. In short, the negative memories that partly constitute ruminative thought may strengthen 
in association and undergo schematization, increasing the readiness with which they come to mind in the future, despite their having little utility for problem solving.

These considerations suggest that rumination may exact a double cost—at once impairing adaptive learning from external cues and modifying negative memories so as to make them more likely to propel rumination in the future. This could lead to a path dependency, whereby an initial slight tendency to ruminate weakens learning and strengthens negative memories in a way that increases the likelihood of future rumination in a similar situation, subsequently leading to greater cost to learning and modification of negative memories, and so on. Future research could connect laboratory investigation of the cost rumination exacts on $\mathrm{RL}$, and its effects on the associative strength and abstraction of memories, to real-world assessment of the disruptive effects of rumination on ongoing behavior (e.g., Webb et al., in press). Another interesting direction is to investigate whether the proposed positive-feedback loop_-borne of increased preoccupation with negative decontextualized memories paired with weaker learning from external contingenciesrelates to the observation that dynamical signatures of positive-feedback loops precede depressive states (e.g., Leemput et al., 2014). Finally, it is important to note that the positivefeedback loop we are proposing is in the opposite direction as one recently postulated to lead to virtuous cycles of reward surprise and escalating mood caused by serotonergic agonists (Michely, Eldar, Martin, \& Dolan, 2020), yet at extremes create oscillatory mood dynamics that could contribute to the symptoms of Bipolar Disorders (Eldar \& Niv, 2015). In short, we propose a positive-feedback loop that predicts real-world dynamics unfolding over time (Hitchcock, Fried, \& Frank, in press), which could be tested in future longitudinal research.

An important caveat to the present findings, however, is that our computational model of learning in the $\mathrm{RL}$ task in this study was unable to pinpoint specific disruptions responsible for the impairment in performance during state rumination. In fact, our modeling results suggest that attentional breadth (over features learned about in the task) and learning from positive and negative prediction errors are not affected by manipulated rumination. However, we note that 
model validation (via simulations) suggested that our models did not learn the task as well as human participants (see Radulescu et al., 2016, study 2, for a similar result), implying the models may be missing a mechanism for faster learning (which may be the one affected by rumination). Although we tried several alternate models (some reported here), this set of models is certainly not exhaustive and we hope future work can reveal this mechanism (task data will be posted online; see "Declarations"). We note that this failure of the model to explain all aspects of task performance also complicates the interpretation of the current parameter estimates, especially the learning rates (Nassar \& Frank, 2016). Thus, while it remains plausible that the performance deficit observed in the RL task arose from a deficit in learning, like Whitmer et al. (2012) we were unable to pinpoint this definitively within our task and model, hence this remains an important direction for future research.

What mechanism might be missing from our computational model? One possibility is working memory (WM)-transient representations of task-relevant information, which are plausibly affected by the dual task of rumination. Whitmer et al. (2012) proposed that WM may have been responsible for the difficulty they observed among induced ruminators in avoiding punishing stimuli in a test phase administered well after an initial learning phase. In support, they noted prior research had provided indirect evidence for a role for WM in the RL task used in their study (Cavanagh et al., 2010; Frank et al., 2007). Long-term difficulty in avoiding punishing stimuli could have arisen if, in the initial learning phase of their task, rumination had resulted in preferential representation of punishment experiences in WM (see Frey \& McCabe, 2020 for a similar suggestion in a social learning task). This suggestion is especially plausible in light of a recent task that distinguishes the contributions of $\mathrm{WM}$ from $\mathrm{RL}$ in learning and decision making (Collins \& Frank, 2012). This task has shown that prediction errors experienced by the RL system are dampened in proportion to the contribution of WM to learning (Collins, Ciullo, Frank, \& Badre, 2017; Collins \& Frank, 2018). Hence, if in Whitmer et al.'s (2012) study the WM system was especially apt to represent punishment contingencies when learning while ruminating, this could 
have "crowded out" the RL system's contribution to learning from these contingencies. In the later test phase-at which point WM-based representations would have decayed, leaving choice to be guided only by whatever had been learned by the RL system-this would lead to an impaired ability to avoid actions previously associated with punishment. In our study, we were unable to test for such a role of WM because we did not have a test phase and we were unable to model WM computationally (see Supplemental Material, available online for details). Thus, learning rates in our model could reflect a mixture of WM and RL (Nassar \& Frank, 2016). And whether state rumination affects $\mathrm{WM}$ contributions to $\mathrm{RL}$, and whether it does so asymmetrically for punishments, remain open questions.

Although we were unable to resolve these questions, our modeling did show that rumination manipulation had no influence on a "decay" parameter, which represents attentional breadth in the RL task. This is surprising because much research implicates rumination in hijacking attentional systems (e.g., Figueroa et al., 2019; Hamilton, Farmer, Fogelman, \& Gotlib, 2015; Hsu \& Davison, 2017). It is thus noteworthy that we found trait rumination (between subjects) was associated with decreased attentional breadth, as per the decay parameter, yet not with impaired performance. These results are consistent with the prominent "attentional-scope model" of trait rumination (Whitmer \& Gotlib, 2013).

As far as we are aware, our results are the first to find support for the attentional-scope model in an $\mathrm{RL}$ context that requires selective attention. The attentional-scope model notes that trait-rumination measures appear to reflect, in part, a cognitive style favoring stability of mental content-leading to stable representations and low susceptibility to distraction, but difficulty updating WM contents and narrowness in the range of content summoned to mind from long-term memory. This cognitive style thus trades off stability for flexibility; it leads to impairment on some tasks, yet enhanced performance on others (e.g., Altimarano, Miyake, \& Whitmer, 2010). Prior work may have missed that a narrower attentional scope sometimes enhances performance 
because trait-rumination measures also tend to include content related to depression (Whitmer \& Gotlib, 2013), which is associated with poor task performance (Snyder, 2013).

Our results are consistent with the predictions of the attentional-scope model for two reasons. First, a computational model parameter representing attentional scope (decay) was higher among those reporting higher trait rumination. In our model, this parameter dictates the rate at which the learned values of features from unchosen stimuli shrink toward 0 . Hence, different decay rates effectively model individual differences in the number of features that participants appear to be learning about and considering in tandem, with higher decay leading to a narrower range of features under consideration. The attentional-scope model proposes that trait-ruminative individuals are characterized by a narrower attentional scope, hence it predicts this finding. The attentional-scope model also predicts that the (limited) content that is considered by those with low attentional scope may be attended to more stably (i.e., with less susceptibility to distraction). Hence, our finding that task performance was not substantially impaired by trait rumination is also broadly consistent with the notion in the attentional-scope model that this cognitive style is a tradeoff (less content, but more stable attention)—-that is, neither intrinsically good nor bad. This is because the complex task employed here at times requires flexibility and a wide breadth of attention (where trait ruminators should be worse) and at other times benefits from stable attention toward the target feature (where trait-ruminative individuals should be better; Whitmer \& Gotlib, 2013). However, the Dimensions Task was not designed to dissociate stability from flexibility and thus an interesting direction for future research would be use an RL task that orthogonalizes these components. This would allow directly testing the attentional-scope model prediction that (after controlling for depression symptoms) trait-ruminative individuals will show better performance in stability-demanding aspects of the task, yet worse performance in flexibilitydemanding aspects.

One puzzle is why it is that, although we found the decay rate was associated with impaired performance between subjects, we did not find evidence for compensatory changes in 
other model parameters among high trait ruminators that could explain why trait ruminators did not show impaired behavior on our task. Here too, we suspect that the unmodeled contributions of WM to the task played a role. Specifically, the attentional-scope model predicts more stable WM representations among trait ruminators (albeit at the cost of lower WM capacity and inhibition of no-longer-relevant representations in WM). As noted, such stable representations could have enhanced task performance (e.g., by allowing better WM maintenance of a few features most likely to be the target). In ongoing work, we are examining directly whether trait rumination is associated with altered WM in the RL-WM task designed by Collins and Frank (2012).

It is also noteworthy that not only trait rumination, but also state rumination, may affect attentional scope through influencing affect/mood (Grol, Hertel, Koster, \& de Raedt, 2015; Rowe, Hirsch, \& Anderson, 2007; Vanlessen, de Raedt, Koster, \& Pourtois, 2016; Whitmer \& Gotlib, 2013), arousal (Mather \& Sutherland, 2011), or the value assigned to currently dominant modes of information processing (Huntsinger, Isbell, \& Clore, 2014).

Finally, it is important to note that we have focused on attentional effects within games of the Dimensions Task because we employed a variant of this task where game changes (indicating that a new feature is the target feature) are clearly signaled. However, given that past research has suggested that rumination not only leads to narrower attention to stimuli within an established context (as noted by the attentional-scope model; Whitmer \& Gotlib, 2013), but also is associated with poorer sensitivity to environmental signals of context (Watkins \& Roberts, 2020), rumination may have impaired performance in our task due to "bleeding in" of learning across games (e.g., misremembering whether a specific feature was already "examined" in the current game, or in the previous game). An interesting direction for future research would be to examine how trait and state rumination influence the ability to detect unsignaled changes to task contingencies in an $\mathrm{RL}$ context in tasks designed to detect differences in this ability (e.g., Browning, Behrens, Jocham, O’Reilly, \& Bishop, 2015; Gagne, Zika, Dayan, \& Bishop, 2021; Huang, Thompson, \& Paulus, 2017; Wilson \& Niv, 2011). 
Six limitations and nuances to this study warrant further discussion. First, a manipulation check measure showed only weak evidence for the differential effectiveness of the rumination versus neutral manipulation. There is some ambiguity about how this result should be interpreted due to the possibility that ours was a poor measure of the manipulation's effectiveness. We created this measure for this study because we were not aware of validated state-rumination measures at the time. Such a measure has since been developed; it asks directly about ruminative thought and has well-validated psychometric properties (Marchetti, Mor, Chiorri, \& Koster, 2018). Other studies have successfully used visual-analogue scales to assess changes after rumination (e.g., Watkins \& Teasdale, 2004; Roberts, Watkins, \& Wills, 2013). Notably, many of the seminal experimental rumination-manipulation studies did not include a manipulation check; we hope our study helps to bring attention to this issue's importance.

Second, despite taking significant steps to adapt the classic Nolen-Hoeksema ruminationmanipulation procedure to this study (see "Methods"), the heterogeneity of effects on participants' behavior suggested the procedure was still less well-controlled than would be ideal (Figure 2A). This could have been due to the cues being too subtle, a subset of participants choosing not to engage with the cues, or it is possible that rumination has relatively weak and/or inconsistent effects on RL (i.e., only affecting a subset of subjects). Future research employing multi-modal methods and/or larger samples may help to stratify low responders to the manipulation to identify more precisely the sources of heterogeneity, as well as to more accurately assess the effects of rumination on $\mathrm{RL}$.

Importantly, it is unknown to what extent our adapted procedure, which cued rumination through cues intermittently interleaved with the task, produces a different effect than the classic Nolen-Hoeksema manipulation which uses a single prolonged manipulation phase. In particular, the latter may lead to a cumulative effect through repeatedly focusing attention on related themes that may not have occurred in the current design given that the attention-demanding Dimensions Task interrupted the sequence of rumination cues. As described in Methods, we judged this 
adaptation critical in light of the need for participants to play many trials of the task to allow stable within-subject task-parameter estimation (as established in study development; Hitchcock et al., 2017). Moreover, we were specifically interested in intermittent brief cueing of rumination amid an ongoing task as an analogy to real-life situations where short bouts of rumination occur during an ongoing external activity (such as ruminating in response to repeated perceived social faux pas while at a social event with colleagues), interrupting the ability to adjust to and learn from external contingencies. However, a limitation of our adaptation is that we did not establish whether it replicated key effects of the Nolen-Hoeksema induction (e.g., increasing negative affect and negative self-referential thinking). Finally, we note that another manipulation from the literaturecueing an unresolved personal goal—has been found to produce persistent effects; this may be a good option for designs such as ours that require engaging in a task for an extended period (Roberts et al., 2013).

To summarize, our design involved both adapting the Nolen-Hoeksema paradigm and using a novel manipulation check. Future research investigating rumination's effects on RL using standardized manipulation checks and/or the original manipulation (possibly in combination with strategies for minimizing the necessary number of trials in the RL task; see next paragraph) could help to paint a more complete picture of the effects of state rumination on RL and the boundary conditions thereon. Another important future direction to elucidate boundary conditions is to examine how other forms of repetitive negative thinking (such as worry) alter RL, given that such constructs overlap substantially with rumination (Ehring et al., 2011; Hallion et al., 2019; Segerstorm et al., 2016).

Third, participants might have been disincentivized from engaging with the rumination cues because they had the opportunity to earn a financial bonus for task performance but no incentive to engage with the rumination cues. We incentivized task performance to encourage engagement throughout the long task (as prior work suggested many trials were needed to stably estimate model parameters; Hitchcock et al., 2017). It may be possible to estimate parameters 
accurately with fewer data using hierarchical Bayesian modeling (Haines et al., 2020; Wiecki, Poland, \& Frank, 2015) and/or adaptive task design (Yang, Pitt, Ahn, \& Myung, 2020).

Fourth, as was true in many of the seminal experimental rumination-manipulation studies, we employed a college-student sample. The effects from such studies have tended to hold in clinical samples (Watkins, 2008), but future research should examine how rumination affects RL in clinical and broader population samples. Related, we did not assess the use of psychotropic medications; this is less of a limitation with our within-subject analyses (because each participant served as their own baseline) but it is possible that the trait-rumination and decay parameter relationship could have arisen due to differences in psychotropic medication across participants.

Fifth, we found lower internal consistency in our trait rumination and depression measures than has typically been reported as well as low correlations between these measures (note the latter may be partially accounted for by restriction of range, given the sample was selected for mild or greater depression symptoms and about $84 \%$ of participants fell in a mild or moderate range). This suggests increased risk of measurement noise in effects involving these measures (the trait rumination-decay relationship, and depression as a covariate and moderator of the manipulation effect), which should therefore be interpreted with caution. A final nuance is that more-depressed participants performed relatively poorly in both experimental phases, whereas less-depressed participants performed comparatively well in the neutral phase but then comparably to the more-depressed participants under rumination manipulation. An implication for rumination-manipulation research is that recruiting participants with higher depression severity does not ensure the manipulation will have larger effects-in fact, the opposite may be true.

In sum, we found that rumination altered reinforcement learning, suggesting that disrupted trial-and-error learning may be an underappreciated pathway through which rumination impairs adaptive action, and raising the possibility that such disruption contributes to poor mental health. We also found evidence that state rumination (experimentally manipulated within-subject) and trait rumination (between-subjects) had distinct effects on our task, with only the latter affecting a 
model parameter quantifying attentional breadth during learning. This study paves the way for translational research examining if impairments to learning during rumination, perhaps in concert with rumination modifying negative memories, predict ineffective behavior in everyday life. In terms of basic clinical science, our study underscores the need to pinpoint the exact mechanisms through which state rumination alters learning, which may in turn suggest interventions to prevent or remediate learning differences caused by rumination that would otherwise spur ineffective behavior. 


\section{Declarations}

\section{Acknowledgements}

Thanks to Ayelet Ruscio for advice on the rumination-manipulation procedure and to Blair Wisco for guidance on adapting it to this study. Thanks to Ashleigh Rutherford for feedback on the manuscript.

\section{Funding.}

$\mathrm{PH}$ was supported by NIMH grant F32MH123055. YN was supported by NIMH grant R01MH119511.

\section{Availability of data and materials}

Code used to produce the results and figures are available at github.com/peterhitchcock/rum_derails_rl. Task data will be posted at nivlab.princeton.edu/data upon publication acceptance.

\section{Authors' contributions}

All authors developed the study concept and design. P.H. and N.R. performed data collection. P.H. performed data analyses in consultation with Y.N. and F.Z. and all authors provided feedback and contributed to the interpretation of the results. P.H. drafted the paper and Y.N. provided critical revisions. All authors approved the final manuscript for submission.

Ethics approval and consent to participate

All participants gave informed consent prior to the study and the study was approved by The Drexel University Institutional Review Board.

\section{Consent for publication}

Not applicable.

\section{Competing interests}

None declared. 


\section{References}

Abela, J. R., Brozina, K., \& Haigh, E. P. (2002). An examination of the response styles theory of depression in third-and seventh-grade children: A short-term longitudinal study. Journal of Abnormal Child Psychology, 30(5), 515-527. PMID: 12403154

Aldao, A., Nolen-Hoeksema, S., \& Schweizer, S. (2010). Emotion-regulation strategies across psychopathology: A meta-analytic review. Clinical psychology review, 30(2), 217-237. PMID: 20015584

Altamirano, L. J., Miyake, A., \& Whitmer, A. J. (2010). When mental inflexibility facilitates executive control: Beneficial side effects of ruminative tendencies on goal maintenance. Psychological Science, 21(10), 1377-1382.

Audrain, S., \& McAndrews, M. P. (2020). Schemas provide a scaffold for neocortical integration at the cost of memory specificity over time (preprint). bioRxiv. https://doi.org/10.1101/2020.10.11.335166

Bates, D., Kliegl, R., Vasishth, S., \& Baayen, H. (2015). Parsimonious mixed models (preprint). arXiv. 1506.04967.

Bates, D., Mächler, M., Bolker, B., \& Walker, S. (2014). Fitting linear mixed-effects models using Ime4 (preprint). arXiv. 1406.5823.

Barr, D. J., Levy, R., Scheepers, C., \& Tily, H. J. (2013). Random effects structure for confirmatory hypothesis testing: Keep it maximal. Journal of memory and language, 68(3), 255-278.

Beck, A. T., Steer, R. A., \& Brown, G. K. (1996). Beck depression inventory-II. San Antonio, 78(2), 490498.

Brown, V. M., Chen, J., Gillan, C. M., \& Price, R. B. (2020). Improving the reliability of computational analyses: Model-based planning and its relationship with compulsivity. Biological Psychiatry: Cognitive Neuroscience and Neuroimaging, , 5(6), 601-609.

Browning, M., Behrens, T. E., Jocham, G., O'reilly, J. X., \& Bishop, S. J. (2015). Anxious individuals have difficulty learning the causal statistics of aversive environments. Nature neuroscience, 18(4), 590596.

Ciesla, J. A., \& Roberts, J. E. (2007). Rumination, negative cognition, and their interactive effects on depressed mood. Emotion, 7(3), 555-565.

Cohen, R. T., \& Kahana, M. J. (2019). Retrieved-context theory of memory in emotional disorders. bioRxiv. (preprint) https://doi.org/10.1101/817486.

Collins, A. G., Ciullo, B., Frank, M. J., \& Badre, D. (2017). Working memory load strengthens reward prediction errors. Journal of Neuroscience, 37(16), 4332-4342.

Collins, A. G., \& Frank, M. J. (2012). How much of reinforcement learning is working memory, not reinforcement learning? A behavioral, computational, and neurogenetic analysis. European Journal of Neuroscience, 35(7), 1024-1035.

Collins, A. G., \& Frank, M. J. (2018). Within-and across-trial dynamics of human EEG reveal cooperative interplay between reinforcement learning and working memory. Proceedings of the National Academy of Sciences, 115(10), 2502-2507. 
Cook, L., Mostazir, M., \& Watkins, E. (2019). Reducing Stress and Preventing Depression (RESPOND): Randomized Controlled Trial of Web-Based Rumination-Focused Cognitive Behavioral Therapy for High-Ruminating University Students. Journal of medical Internet research, 21(5), e11349. PMC6536298

Daniel, R., Radulescu, A., \& Niv, Y. (2020). Intact reinforcement learning but impaired attentional control during multidimensional probabilistic learning in older adults. Journal of Neuroscience, 40(5), 1084-1096.

Eldar, E., \& Niv, Y. (2015). Interaction between emotional state and learning underlies mood instability. Nature communications, 6(1), 1-10.

Ehring, T., Zetsche, U., Weidacker, K., Wahl, K., Schönfeld, S., \& Ehlers, A. (2011). The Perseverative Thinking Questionnaire (PTQ): Validation of a content-independent measure of repetitive negative thinking. Journal of behavior therapy and experimental psychiatry, 42(2), 225-232.

Feurer, C., Burkhouse, K. L., Siegle, G., \& Gibb, B. E. (2017). Increased pupil dilation to angry faces predicts interpersonal stress generation in offspring of depressed mothers. Journal of Child Psychology and Psychiatry, 58(8), 950-957. PMC5513778

Figueroa, C. A., Cabral, J., Mocking, R. J., Rapuano, K. M., van Hartevelt, T. J., Deco, G., ... \& Ruhé, H. G. (2019). Altered ability to access a clinically relevant control network in patients remitted from major depressive disorder. Human brain mapping.

Fried, E. I., \& Nesse, R. M. (2015). Depression sum-scores don't add up: why analyzing specific depression symptoms is essential. BMC medicine, 13(1), 1-11.

Gagne, C., Zika, O., Dayan, P., \& Bishop, S. J. (2020). Impaired adaptation of learning to contingency volatility in internalizing psychopathology. Elife, 9, e61387.

Gelman, A. (2018). The failure of null hypothesis significance testing when studying incremental changes, and what to do about it. Personality and Social Psychology Bulletin, 44(1), 16-23.

Grant, D. M., Mills, A. C., Judah, M. R., \& White, E. J. (2019). State and trait effects of rumination on inhibitory processes in memory. Current Psychology, 1-9.

Grimbos, T., Granic, I., \& Pepler, D. (2013). The relation between co-rumination, maternal depressive symptoms and child psychopathology. Journal of psychopathology and behavioral assessment, 35(3), 335-345.

Haines, N., Kvam, P. D., Irving, L. H., Smith, C., Beauchaine, T. P., Pitt, M. A., Ahn, W., \& Turner, B. (2020). Learning from the Reliability Paradox: How Theoretically Informed Generative Models Can Advance the Social, Behavioral, and Brain Sciences (preprint). PsyArXiv. https://doi.org/10.31234/osf.io/xr7y3

Hallion, L. S., Wright, A. G., Joormann, J., Kusmierski, S. N., Coutanche, M. N., \& Caulfield, M. K. (2019, April 1). A Five Factor Model of Perseverative Thought. https://doi.org/10.31234/osf.io/gu7xp

Hamilton, J. P., Farmer, M., Fogelman, P., \& Gotlib, I. H. (2015). Depressive rumination, the defaultmode network, and the dark matter of clinical neuroscience. Biological psychiatry, 78(4), 224-230.

Hamilton, J. P., Furman, D. J., Chang, C., Thomason, M. E., Dennis, E., \& Gotlib, I. H. (2011). Defaultmode and task-positive network activity in major depressive disorder: implications for adaptive and maladaptive rumination. Biological psychiatry, 70(4), 327-333. 
Hayes, S. C., Strosahl, K. D., \& Wilson, K. G. (2011). Acceptance and commitment therapy: The process and practice of mindful change. New York: Guilford Press.

Herbert, J., Forman, E., \& Hitchcock, P. (2016). Contextual approaches to psychotherapy: Defining, distinguishing, and common features. In R. Zettle, S. C. Hayes, D. Barnes-Holmes, \& A. Biglan (Eds.). The Wiley handbook of contextual behavioral science (pp. 287-302). Oxford, UK: Blackwell Publishing Ltd.

Hertel, P. T., \& Rude, S. S. (1991). Depressive deficits in memory: Focusing attention improves subsequent recall. Journal of Experimental Psychology: General, 120(3), 301-309.

Hitchcock, P., Ph.D., Fried, E. I., \& Frank, M. (in press). Computational Psychiatry Needs Time and Context. Annual Reviews of Psychology, 73, https://doi.org/10.1146/annurev-psych-021621124910 [https://psyarxiv.com/xf2u3]

Hitchcock, P., Radulescu, A., Niv, Y., Rothstein, N., Sims, C. (2017). Translating a Reinforcement Learning Task into a Computational Psychiatry Assay: Challenges and Strategies. Proceedings of the 39th Annual Meeting of the Cognitive Science Society.

Hsu, K. J., \& Davison, G. C. (2017). Compounded deficits: The association between neuropsychological impairment and attention biases in currently depressed, formerly depressed, and never depressed individuals. Clinical Psychological Science, 5(2), 286-298.

Huang, H., Thompson, W., \& Paulus, M. P. (2017). Computational dysfunctions in anxiety: Failure to differentiate signal from noise. Biological psychiatry, 82(6), 440-446.

Jones, N. P., Siegle, G. J., Muelly, E. R., Haggerty, A., \& Ghinassi, F. (2010). Poor performance on cognitive tasks in depression: Doing too much or not enough? Cognitive, Affective, \& Behavioral Neuroscience, 10(1), 129-140. PMC2841800

Kaiser, R. H., Snyder, H. R., Goer, F., Clegg, R., Ironside, M., \& Pizzagalli, D. A. (2018). Attention bias in rumination and depression: Cognitive mechanisms and brain networks. Clinical Psychological Science, 6(6), 765-782.

Kuehner, C., \& Weber, I. (1999). Responses to depression in unipolar depressed patients: An investigation of Nolen-Hoeksema's response styles theory. Psychological medicine, 29(6), 13231333. PMID: 10616938

Kuhl, J. (1981). Motivational and functional helplessness: The moderating effect of state versus action orientation. Journal of personality and social psychology, 40(1), 155-170.

van de Leemput, I. A., Wichers, M., Cramer, A. O., Borsboom, D., Tuerlinckx, F., Kuppens, P., ... \& Scheffer, M. (2014). Critical slowing down as early warning for the onset and termination of depression. Proceedings of the National Academy of Sciences, 111(1), 87-92.

Lehtonen, A., Jakub, N., Craske, M., Doll, H., Harvey, A., \& Stein, A. (2009). Effects of preoccupation on interpersonal recall: A pilot study. Depression and anxiety, 26(1), 1-6.

Leong, Y. C., Radulescu, A., Daniel, R., DeWoskin, V., \& Niv, Y. (2017). Dynamic interaction between reinforcement learning and attention in multidimensional environments. Neuron, 93(2), 451-463.

Lewis, E. J., Blanco, I., Raila, H., \& Joormann, J. (2019). Does repetitive negative thinking affect attention? Differential effects of worry and rumination on attention to emotional stimuli. Emotion, $19(8), 1450-1462$. 
Lyubomirsky, S., Layous, K., Chancellor, J., \& Nelson, S. K. (2015). Thinking about rumination: the scholarly contributions and intellectual legacy of Susan Nolen-Hoeksema. Annual Review of Clinical Psychology, 11, 1-22.

Lyubomirsky, S., \& Nolen-Hoeksema, S. (1993). Self-perpetuating properties of dysphoric rumination. Journal of personality and social psychology, 65(2), 339-349.

Lyubomirsky, S., \& Tkach, C. (2004). The consequences of dysphoric rumination. In C. Papageorgiou \& A. Wells (Eds.), Depressive rumination: Nature, theory, and treatment (pp. 21-42). New York: Wiley.

Maia, T. V., \& Frank, M. J. (2011). From reinforcement learning models to psychiatric and neurological disorders. Nature neuroscience, 14(2), 154-162.

Marchetti, I., Mor, N., Chiorri, C., \& Koster, E. H. (2018). The brief state rumination inventory (BSRI): validation and psychometric evaluation. Cognitive Therapy and Research, 42(4), 447-460.

Martell, C. R., Dimidjian, S., \& Herman-Dunn, R. (2013). Behavioral activation for depression: A clinician's guide (pp. 129-148). New York: Guilford Press.

Matuschek, H., Kliegl, R., Vasishth, S., Baayen, H., \& Bates, D. (2017). Balancing Type I error and power in linear mixed models. Journal of Memory and Language, 94, 305-315.

Mirman, D. (2017). Growth curve analysis and visualization using R. Chapman and Hall/CRC: Boca Raton, FL.

McLaughlin, K. A., Aldao, A., Wisco, B. E., \& Hilt, L. M. (2014). Rumination as a transdiagnostic factor underlying transitions between internalizing symptoms and aggressive behavior in early adolescents. Journal of abnormal psychology, 123(1), 13-23. PMC4222508

McLaughlin, K. A., Borkovec, T. D., \& Sibrava, N. J. (2007). The effects of worry and rumination on affect states and cognitive activity. Behavior Therapy, 38, 23-38.

McLaughlin, K. A., \& Nolen-Hoeksema, S. (2012). Interpersonal stress generation as a mechanism linking rumination to internalizing symptoms in early adolescents. Journal of Clinical Child \& Adolescent Psychology, 41(5), 584-597. PMC3465705

McLaughlin, K. A., \& Nolen-Hoeksema, S. (2011). Rumination as a transdiagnostic factor in depression and anxiety. Behaviour research and therapy, 49(3), 186-193. PMC4222508

Michely, J., Eldar, E., Martin, I. M., \& Dolan, R. J. (2020). A mechanistic account of serotonin's impact on mood. Nature communications, 11(1), 1-11.

Moberly, N. J., \& Watkins, E. R. (2006). Processing mode influences the relationship between trait rumination and emotional vulnerability. Behavior Therapy, 37(3), 281-291.

Moberly, N. J., \& Watkins, E. R. (2010). Negative affect and ruminative self-focus during everyday goal pursuit. Cognition \& Emotion, 24(4), 729-739.

Morey, R. D., Rouder, J. N., \& Jamil, T. (2018). Package 'BayesFactor'. Retrieved from https://cran.rproject.org/web/packages/BayesFactor/BayesFactor.pdf

Moulds, M. L., Kandris, E., Starr, S., \& Wong, A. C. (2007). The relationship between rumination, avoidance and depression in a non-clinical sample. Behaviour research and therapy, 45(2), 251261. PMID: 16631110 
Nassar, M. R., \& Frank, M. J. (2016). Taming the beast: extracting generalizable knowledge from computational models of cognition. Current opinion in behavioral sciences, 11, 49-54.

National Institutes of Mental Health. (2015). The Anatomy of NIMH Funding. Retrieved from http://www.nimh.nih.gov/funding/funding-strategy-for-research-grants/white-paper_149362.pdf

Niv, Y. (2019). Learning task-state representations. Nature neuroscience, 22(10), 1544-1553.

Niv, Y., Daniel, R., Geana, A., Gershman, S. J., Leong, Y. C., Radulescu, A., \& Wilson, R. C. (2015). Reinforcement learning in multidimensional environments relies on attention mechanisms. Journal of Neuroscience, 35(21), 8145-8157.

Nolen-Hoeksema, S. (1987). Sex differences in unipolar depression: evidence and theory. Psychological bulletin, 101(2), 259-282. PMID: 3562707

Nolen-Hoeksema, S. (1991). Responses to depression and their effects on the duration of depressive episodes. Journal of abnormal psychology, 100(4), 569-582. PMID: 1757671

Nolen-Hoeksema, S., \& Morrow, J. (1993). Effects of rumination and distraction on naturally occurring depressed mood. Cognition \& Emotion, 7(6), 561-570.

Nolen-Hoeksema, S., Wisco, B. E., \& Lyubomirsky, S. (2008). Rethinking rumination. Perspectives on psychological science, 3(5), 400-424. PMID: 26158958

Paulus, M. P., Huys, Q. J., \& Maia, T. V. (2016). A roadmap for the development of applied computational psychiatry. Biological psychiatry: cognitive neuroscience and neuroimaging, 1(5), 386-392.

Pearson, K. A., Watkins, E. R., Mullan, E. G., \& Moberly, N. J. (2010). Psychosocial correlates of depressive rumination. Behaviour Research and Therapy, 48(8), 784-791. PMID: 20538263

Philippot, P., \& Brutoux, F. (2008). Induced rumination dampens executive processes in dysphoric young adults. Journal of behavior therapy and experimental psychiatry, 39(3), 219-227.

Radulescu, A., Daniel, R., \& Niv, Y. (2016). The effects of aging on the interaction between reinforcement learning and attention. Psychology and aging, 31(7), 747-757.

Radulescu, A., Niv, Y., \& Ballard, I. (2019). Holistic reinforcement learning: the role of structure and attention. Trends in cognitive sciences, 23(4), 278-292.

Reilly, E. E., Lavender, J. M., Berner, L. A., Brown, T. A., Wierenga, C. E., \& Kaye, W. H. (2019). Could repetitive negative thinking interfere with corrective learning? The example of anorexia nervosa. International Journal of Eating Disorders, 52(1), 36-41.

Roberts, H., Watkins, E. R., \& Wills, A. J. (2013). Cueing an unresolved personal goal causes persistent ruminative self-focus: An experimental evaluation of control theories of rumination. Journal of Behavior Therapy and Experimental Psychiatry, 44(4), 449-455.

Rmus, M., McDougle, S. D., \& Collins, A. G. (2021). The role of executive function in shaping reinforcement learning. Current Opinion in Behavioral Sciences, 38, 66-73.

Rodebaugh, T. L., Scullin, R. B., Langer, J. K., Dixon, D. J., Huppert, J. D., Bernstein, A., ... \& Lenze, E. J. (2016). Unreliability as a threat to understanding psychopathology: The cautionary tale of attentional bias. Journal of Abnormal Psychology, 125(6), 840-851. 
Rouder, J. N., Speckman, P. L., Sun, D., Morey, R. D., \& Iverson, G. (2009). Bayesian t tests for accepting and rejecting the null hypothesis. Psychonomic bulletin \& review, 16(2), 225-237.

Schwarz, G. (1978). Estimating the dimension of a model. Annals of Statistics, 6, 461-464.

Segerstrom, S. C., Hardy, J. K., Evans, D. R., Boggero, I. A., Alden, L. E., \& Stanton, A. L. (2016). Briefly assessing repetitive thought dimensions: Valence, purpose, and total. Assessment, 23(5), 614623.

Smarr, K. L., \& Keefer, A. L. (2011). Measures of depression and depressive symptoms: Beck Depression Inventory-II (BDI-II), Center for Epidemiologic Studies Depression Scale (CES-D), Geriatric Depression Scale (GDS), Hospital Anxiety and Depression Scale (HADS), and Patient Health Questionnaire-9 (PHQ9). Arthritis care \& research, 63(S11), S454-S466.

Snyder, H. R., \& Hankin, B. L. (2016). Spiraling out of control: Stress generation and subsequent rumination mediate the link between poorer cognitive control and internalizing psychopathology. Clinical psychological science, 4(6), 1047-1064. PMC5102509

Snyder, H. R. (2013). Major depressive disorder is associated with broad impairments on neuropsychological measures of executive function: a meta-analysis and review. Psychological bulletin, 139(1), 81-132.

Spasojević, J., \& Alloy, L. B. (2001). Rumination as a common mechanism relating depressive risk factors to depression. Emotion, 1(1), 25-37. PMID: 12894809

Stein, A., Craske, M. G., Lehtonen, A., Harvey, A., Savage-McGlynn, E., Davies, B., . . Counsell, N. (2012). Maternal cognitions and mother-infant interaction in postnatal depression and generalized anxiety disorder. Journal of Abnormal Psychology, 121(4), 795-809. doi:10.1037/a0026847

Stein, A., Lehtonen, A., Harvey, A. G., Nicol-Harper, R., \& Craske, M. (2009). The influence of postnatal psychiatric disorder on child development. Psychopathology, 42(1), 11-21.

Sutton, R. S., \& Barto, A. G. (2018). Reinforcement learning: An introduction. Cambridge, MA: MIT press.

Tester-Jones, M., Karl, A., Watkins, E., \& O'Mahen, H. (2017). Rumination in dysphoric mothers negatively affects mother-infant interactions. Journal of Child Psychology and Psychiatry, 58(1), 38-45. PMID: 27616434

Titov, N., Dear, B. F., McMillan, D., Anderson, T., Zou, J., \& Sunderland, M. (2011). Psychometric comparison of the PHQ-9 and BDI-II for measuring response during treatment of depression. Cognitive Behaviour Therapy, 40(2), 126-136.

Treynor, W., Gonzalez, R., \& Nolen-Hoeksema, S. (2003). Rumination reconsidered: A psychometric analysis. Cognitive therapy and research, 27(3), 247-259.

Van Vugt, M. K., Hitchcock, P., Shahar, B., \& Britton, W. (2012). The effects of mindfulness-based cognitive therapy on affective memory recall dynamics in depression: a mechanistic model of rumination. Frontiers in human neuroscience, 6, 257.

Van Vugt, M. K., van der Velde, M., \& ESM-MERGE Investigators. (2018). How does rumination impact cognition? A first mechanistic model. Topics in Cognitive Science, 10(1), 175-191. 
Walker, E. R., McGee, R. E., \& Druss, B. G. (2015). Mortality in mental disorders and global disease burden implications: a systematic review and meta-analysis. JAMA psychiatry, 72(4), 334-341.

Watkins, E. R. (2008). Constructive and unconstructive repetitive thought. Psychological bulletin, 134(2), 163-206. PMC2672052

Watkins, E. R., \& Roberts, H. (2020). Reflecting on rumination: Consequences, causes, mechanisms and treatment of rumination. Behaviour Research and Therapy, 127, 103573.

Watkins, E., \& Teasdale, J. D. (2004). Adaptive and maladaptive self-focus in depression. Journal of affective disorders, 82(1), 1-8.

Webb, C. A., Israel, E. S., Belleau, E., Appleman, L., Forbes, E. E., \& Pizzagalli, D. A. (In Press). MindWandering in Adolescents Predicts Worse Affect and Is Linked to Aberrant Default Mode Network-Salience Network Connectivity. Journal of the American Academy of Child \& Adolescent Psychiatry.

Wiecki, T. V., Poland, J., \& Frank, M. J. (2015). Model-based cognitive neuroscience approaches to computational psychiatry: clustering and classification. Clinical Psychological Science, 3(3), 378399.

Williams, J. M. G. (1996). Depression and the specificity of autobiographical memory. In D. C. Rubin (Ed.), Remembering our past: Studies in autobiographical memory (pp. 244-267). Cambridge, England: Cambridge University Press.

Whitmer, A. J., Frank, M. J., \& Gotlib, I. H. (2012). Sensitivity to reward and punishment in major depressive disorder: effects of rumination and of single versus multiple experiences. Cognition \& emotion, 26(8), 1475-1485.

Whitmer, A. J., \& Gotlib, I. H. (2013). An attentional scope model of rumination. Psychological Bulletin, 139(5), 1036-1061.

Wilson, R. C., \& Niv, Y. (2012). Inferring relevance in a changing world. Frontiers in human neuroscience, 5, 189.

Yang, J., Pitt, M. A., Ahn, W. Y., \& Myung, J. I. (2020). ADOpy: a python package for adaptive design optimization. Behavior Research Methods, 1-24. 


\section{Supplementary Materials}

\section{Methodological Details S1.}

\section{Manipulation Checks}

The following list gives all items shown during manipulation checks. For each item, the prompt "Use the scale below to describe yourself RIGHT NOW." was shown at the top of the

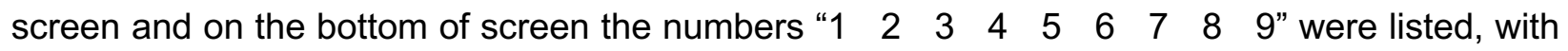
the item on the left below the 1 (e.g., "Not sad" for 1.) and the item on the right below the 9 (e.g., "Very sad" for 1.). Participants entered a number to proceed to the next screen. Note that the italicized items had the position of the more extreme anchor reversed to promote careful responding by participants (these were re-coded during analysis such that higher numbers always meant the more extreme emotion).

1. Not sad; Very sad

2. Not angry; Very angry

3. Very happy; Not happy

4. Very anxious; Not anxious

5. Not depressed; Very depressed

6. Very content; not content

7. Very joyful; not joyful

8. Very worried; not worried

9. Not brooding; Brooding

10. Focused on myself; Not focused on myself

11. Critical of myself; Not critical of myself

\section{Computational Modeling}

Model fitting. Best-fitting parameter estimates for each participant were obtained via maximum likelihood estimation (Daniel et al., 2020; Niv et al., 2015; Radulescu et al., 2016) implemented by minimizing the negative log likelihood of each participant's observed choices using the optim function in base R. Intuitively, this procedure works as follows. The likelihood that a given participant would make their empirically observed choice on a given trial, given one of the models described in the main text and a specific setting of parameters (e.g., $\theta_{M=f R L+\text { decay }}=\{\eta=.1$, $d=.4, \beta=5\}$ ), can be calculated by finding the probability of choosing that stimulus from equation 4 (main text) conditional on their learning history up to that point. Hence, the likelihood of the 
participant's full set of choices (under the model and parameter settings) can be estimated as the product of the likelihoods of those choices:

$$
L=p\left(C_{1: T} \mid \theta_{M}\right)=\prod_{t=1}^{T} p\left(C_{t} \mid D_{1: t-1}, \theta_{M}\right)
$$

where $C$ represents choice, $\theta_{M}$ represents the parameters of a given model, $T$ is the number of trials, and $D_{1: t-1}$ represents the data observed on all trials prior to the current one. The process of searching through the parameter space to find the configuration of parameters producing the highest likelihood is known as maximum likelihood estimation and is done by minimizing the negative of the likelihood function as a function of the model parameters. In practice, the product in equation 5 (main text) produces very small values (due to multiplying many values less than 0 ) hence we minimized the negative log of the likelihood (which is monotonic in $L$ and is less subject to machine precision errors).

Model Comparison. For selecting which model described the data best, we used the Bayesian Information Criterion (BIC; Schwarz, 1978) approximation of model evidence, $E_{M}$, estimated as,

$$
E_{M}=-2 \log \left(p\left(D \mid M, \hat{\theta}_{M}\right)\right)+\left\|\hat{\theta}_{M}\right\| \log T
$$

In this equation, $-\log \left(p\left(D \mid M, \hat{\theta}_{M}\right)\right.$ represents the negative log likelihood of the data given the model and the best-fit parameters $\hat{\theta}$, and $\left\|\hat{\theta}_{M}\right\| \log T$ is a penalty term that increases with the number of parameters of the model $\|\hat{\theta}\|$ and the number of trials $T$ thus helping to prevent overfitting by overly complex models. A lower BIC score corresponds to a better model of the data.

Critically, to determine the computational effects of the within-subject manipulation of RUM vs. NEU, we used the BIC to determine if participants' data were best explained by varying each model parameter across conditions or using the same parameter value across conditions. We tested this separately for each parameter; no parameter justified splitting hence combinations of splits were not tested. To appropriately penalize the split models for greater complexity via the second term in equation 5, we counted split parameters as two parameters, whereas non-split parameters were counted as a single parameter, and $T$ was adjusted to the number of trials to which each parameter was fit (i.e., 1320 for a parameter fit to the full dataset and 660 for a parameter fit to a single induction condition). The same results held when using a likelihood ratio test for nested models and requiring significant improvement for a majority of subjects at $\alpha=.05$ to justify additional parameters. 
Model Simulations and Parameter Recovery. Model simulations were performed by using participant's best-fitting parameters $\hat{\theta}$ with the corresponding model and simulating trajectories through the task using the same task contingencies as participants experienced, but without using participants' actual choices and rewards. The ability to recover model parameters was assessed by simulating the same number of trials on which the models were empirically fit using parameters in the range of the empirical data and examining how well our maximum likelihood procedure could recover the true parameters that had generated the simulated data.

Attempts to Model Working Memory. We attempted to model the contribution of working memory to the Dimensions Task, but all models we considered showed unacceptable parameter identifiability. This parallels the experience of Collins, Frank, and colleagues, who found it difficult to model working memory in standard reinforcement learning tasks, thus motivating them to develop the working memory and reinforcement learning task discussed in the main text (Frank, personal communication). Unlike the task in the current study, their task has deterministic rather than stochastic contingencies (as well as other features) that increase the ability to dissociate RL from WM. 
Table S1. Results of manipulation check mixed-effects model.

\begin{tabular}{lllcc}
\hline & $b$ & $\mathrm{SE}$ & $t$ & $p$ \\
\hline Intercept & 4.88 & .18 & 26.91 & $<.001^{* *}$ \\
Condition: $\mathrm{RUM}=1, \mathrm{NEU}=0$ & .28 & .14 & 1.99 & $.063^{\dagger}$ \\
Baseline score (z-scored) & 1.21 & .12 & 10.01 & $<.001^{* *}$ \\
First condition administered: RUM $=1 ; \mathrm{NEU}=0$ & -.04 & .18 & -0.20 & .840 \\
\hline Note: Fixed effects. ${ }^{* *} p<.01,{ }^{*} p<.05,{ }^{\dagger} p<.10$ & & & & \\
\hline
\end{tabular}

\begin{tabular}{ll}
\hline Manipulation check item & Slope \\
\hline Depressed & .27 \\
Critical of myself & .21 \\
Focused on myself & .21 \\
Worried & .15 \\
Sad & .05 \\
Angry & -.03 \\
Anxious & -.14 \\
Happy (reverse scored) & -.18 \\
Brooding & -.23 \\
Joyful (reverse scored) & -.29 \\
Content (reverse scored) & -.38 \\
\hline
\end{tabular}

Note: Item-level random effects for condition. 


\section{Other Materials (not included in publication)}

\section{Manipulation Cues}

Note: Cues developed by Nolen-Hoeksema and colleagues

Rumination Manipulation

\section{Think About:}

1. the physical sensations you feel in your body

2. your character and who you strive to be

3. the degree of clarity in your thinking right now

4. why you react the way you do

5. the way you feel inside

6. the possible consequences of your current mental state

7. how similar/different you are relative to other people

8. what it would be like if your present feelings lasted

9. why things turn out the way they do

10. trying to understand your feelings

11. how awake/tired you feel now

12. the amount of tension in your muscles

13. whether you are fulfilled

14. your physical appearance

15. whether you feel stressed right now

16. the long-term goals you have set

17. the amount of certainty you feel

18. your present feelings of fatigue/energy

19. possible explanations for your physical sensations

20. how hopeful/hopeless you are feeling 
21. the level of motivation you feel right now

22. the degree of helplessness you feel

23. the degree of calmness/restlessness you feel

24. the possible consequences of the way you feel

25. what your feelings might mean

26. how sad/happy you are feeling

27. the expectations your family has for you

28. why your body feels this way

29. why you get this way sometimes

30. how passive/active you feel

31. what people notice about your personality

32. the kind of student you are and wish you were

33. how weak/strong your body feels now

34. the degree of relaxation/agitation you feel

35. the kind of person you think you should be

36. the degree of control you feel right now

37. what would happen if your current physical state lasted

38. sitting down and analyzing your personality

39. why you turned out this way

40. the things that are most important in your life

41. whether you have accomplished a lot so far

42. the degree of decisiveness you feel

43. trying to understand who you are

44. how you feel about your friendships 


\section{Distraction Manipulation}

\section{Think About:}

1. and imagine a boat slowly crossing the Atlantic

2. the layout of a typical classroom

3. the shape of a large black umbrella

4. the movement of an electric fan on a warm day

5. raindrops sliding down a windowpane

6. a double-decker bus driving down a street

7. and picture a full moon on a clear night

8. clouds forming in the sky

9. the layout of the local shopping center

10. and imagine a plane flying overhead

11. fire darting around a log in a fireplace

12. and concentrate on the expression on the face of the Mona Lisa

13. a clown putting on his or her make-up

14. two birds sitting on a tree branch

15. the shadow of a stop sign

16. the layout of the local post office

17. the structure of a high-rise office building

18. and picture the Eiffel Tower

19. and imagine a truckload of watermelons

20. the pattern on an Oriental rug

21. the "man in the moon"

22. the shape of the continent of Africa

23. a band playing outside

24. a group of polar bears fishing in a stream

25. the shape of the torch on the Statue of Liberty

26. the shape of your home state 
27. the way the ocean looks at sunset

28. the layout of Drexel's campus

29. a train stopped at a station

30. a lone cactus in the desert

31. the shape of the country of Italy

32. a row of shampoo bottles on display

33. a gas station on the side of a highway

34. the fuzz on the shell of a coconut

35. the Presidents' faces on Mount Rushmore

36. and picture Mickey Mouse's face

37. a band playing "The Star Spangled Banner"

38. the shape of a cello

39. the layout of a computer keyboard

40. the shape of the United States

41. the baggage claim area at the airport

42. the shape of a tuba

43.the shape of a baseball glove

44. a freshly painted door 